SUSX-TH-98-01

hep-th/9802099

February 1998

\title{
The Master Equation for the Prepotential
}

\author{
Christos Kokorelis ${ }^{1}$ \\ Centre of Theoretical Physics \\ Sussex University \\ Falmer, Brighton, BN1 9QH, United Kingdom
}

\begin{abstract}
The perturbative prepotential and the Kähler metric of the vector multiplets of the $\mathrm{N}=2$ effective low-energy heterotic strings is calculated directly in $N=1$ sixdimensional toroidal compactifications of the heterotic string vacua. This method provides the solution for the one loop correction to the $\mathrm{N}=2$ vector multiplet prepotential for compactifications of the heterotic string for any rank three and four models, as well for compactifications on $K_{3} \times T^{2}$. In addition, we complete previous calculations, derived from string amplitudes, by deriving the differential equation for the third derivative of the prepotential with respect of the usual complex structure $\mathrm{U}$ moduli of the $T^{2}$ torus. Moreover, we calculate the one loop prepotential, using its modular properties, for $\mathrm{N}=2$ compactifications of the heterotic string exhibiting modular groups similar with those appearing in $\mathrm{N}=2$ sectors of $\mathrm{N}=1$ orbifolds based on non-decomposable torus lattices and on $\mathrm{N}=2$ supersymmetric Yang-Mills.
\end{abstract}

${ }^{1}$ E-mail: c.kokorelis@sussex.ac.uk 


\section{Introduction}

One of the most important aspects of string dualities involve comparisons of the effective actions between $N=2$ compactifications of the ten dimensional heterotic string to lower dimensions and type II superstrings. A key future for testing these dualities is the use of prepotential, which describes the $N=2$ effective low energy theory of vector multiplets in a general supergravity theory.

Guidance in working, with the vector multiplet heterotic prepotential at the string theory level, comes form similar results from $N=2$ supersymmetric Yang-Mills [1]. At the level of $N=2$ supersymmetric $S U(r+1)$ Yang-Mills the quantum moduli space was associated [2] with a particular genus r Riemann surface parametrized by r complex moduli and $2 \mathrm{r}$ periods $\left(\alpha_{D_{i}}, \alpha\right)$. When matter is not present it allows for generic values of the scalar field of the theory to be broken down to the Cartran sub-algebra and it is described from r $N=2$ abelian vector supermultiplets. The theory is dominated from the behaviour of the holomorphic function $\mathcal{F}(\mathcal{A})$, namely the prepotential. The supersymmetric non-linear $\sigma$-model is described by the Kähler potential $K(A, \bar{A})=\operatorname{Im}\left\{\frac{\partial \mathcal{F}(A)}{\partial A} \bar{A}\right\}$, while the metric in its moduli space $\operatorname{Im}(\tau(A))=\operatorname{Im}\left\{\partial^{2} \mathcal{F} / \partial A^{2}\right\}$ is connected to the complexified variable $\theta_{\text {eff }} / \pi+8 \pi i\left(g_{\text {eff }}^{-2}\right) \equiv \tau(A)$. The metric is connected[to the interpretation of the periods $\pi$

$$
\pi=\left(\begin{array}{c}
\alpha_{D}^{i} \\
\alpha^{i}
\end{array}\right), \quad \alpha_{D}^{i}=\frac{\partial \mathcal{F}}{\partial \alpha^{i}}, i=1, \ldots, r
$$

as an appropriate family of a meromorphic one-forms associated with $\lambda$,

$$
\alpha_{D}^{i}=\oint_{\alpha_{i}} \lambda, \quad \alpha^{i}=\oint_{\beta_{i}} \lambda, \quad \tau=\frac{d \alpha_{D}^{i} / d u}{d \alpha_{i} / d u} .
$$

Here, $\alpha_{i}, \beta_{i}$ form a basisf of the homology cycles of the hyperelliptic curve which has the same moduli space as $N=2$ supersymmetric Yang-Mills theory. This means that the metric on the moduli space of supersymmetric Yang-Mills is identified with the period matrix of the hyperelliptic curve. We have to notice that the gauge group is always abelian and the classical enhancement symmetry point is absent from the theory. Only the weak coupling

\footnotetext{
${ }^{1} \alpha$ is the scalar component of the superfield $\mathcal{A}$ and can play the role of the Higgs field.

${ }^{2}$ The cycles $\alpha, \beta$ form a basis of the first homology group $H_{1}\left(E_{g}, Z\right)=Z^{2 g}$, where $E_{g}$ a Riemann surface at genus $g$. The intersection of cycles in the canonical basis takes the form $\left(a_{i}, b_{j}\right)=-\left(b_{j}, a_{i}\right)=\delta_{i j}$.
} 
point is included in the theory, making perturbative calculations for supersymmetric YangMills reliable only in this area of moduli space. The general picture emerging from the study of the supersymmetric Yang-Mills is that the vacuum expectation values of the Higgs fields break the theory to its maximal abelian subgroup. In addition, the exact theory is always in the Higgs phase missing the perturbative point where the non-abelian gauge symmetry is restored.

Lets us now return in string theory to see, how the picture of supersymmetric YangMills is modified and moreover, its connection to the prepotential and the one loop Kähler metric in $N=2$ compactifications of the heterotic string. Various equivalences between the different theories have been proposed and the picture emerging is that the different string theories are expansions of a more fundamental theory around different points in the moduli space of string vacua. We mention string-string duality, where type IIA compactified on $K_{3}$ manifold with $N=4$ supersymmetry has the same moduli space as the heterotic string on a $T^{4}$ torus 51, 52, 53, 54, 48, 50 with $N=4$ supersymmetry. Now the strong coupling of the heterotic string is mapped into the weak coupling of IIA. For $N=2$ type IIB the string generalization of Seiberg-Witten's(SW) quantum theory is provided by the conifold transitions of wrapped three-branesf on Calabi-Yau spaces. Type IIB in ten dimensions admits extremal black holes solutions in the RR sector of the theory. They represent BPS saturated p-brane solitons. Compactification of type IIB on a Calabi-Yau space produces $h^{(1,1)}+1$ supermultiplet moduli with +1 associated with the dilaton and $h^{(2,1)}$ vector multiplets. In addition, it gives the abelian gauge group $U(1)^{h^{(2,1)}+1}$. In general special geometry applied to the compactification of type IIB on the Calabi-Yau space in four dimensions, requires that the scalar component $\mathrm{Z}$ and the prepotential $\mathrm{F}$ of the vector multiplets to be given by the period of the three form $\Omega$ over the canonical homology cycles $a_{I}, b_{I}$ as

$$
Z_{I}=\int_{a_{I}} \Omega, \quad \frac{\partial F}{\partial Z_{I}}=\int_{b_{I}} \Omega, \quad I=1, \ldots, h^{(2,1)} .
$$

Here, $\Omega$ is the holomorphic three form describing the complex structure of the Calabi-

\footnotetext{
${ }^{3}$ The exact effective low energy coupling constant for this theory have been calculated using mirror symmetry.

${ }^{4}$ The three brane of the type IIB can end 47 on the RR five-brane.

${ }^{5}$ See next section.
} 
Yau space. BPS states are $\propto\left|-\hat{\nu}_{e}^{I} Z_{I}+\hat{\nu}_{m}^{I} F_{I}\right|$. The integers $\hat{\nu}_{e}, \hat{\nu}_{m}$ are the electric and magnetic charges of the threebrane wrapped around the three surfaces $a_{I}, b_{I}$. The appearance of a logarithmic singularity in the Kähler metric at the conifold point $Z=0$, involved in the compactification of type IIB on the Calabi-Yau space, is then identified[40] with the extremal three brane black hole becoming massless. In analogy with SW theory, the three-brane becomes massless when the associated cycles vanish. The appearance of the singularity, when the corresponding 3-cycles along the 3-surfaces vanish, is then identified with the existence 40 of a massless black hole solution in the metric of type IIB for the 3-brane. However now, the singularity is not a weak coupling singularity but a strong coupling singularity. This is the analog of Seiberg-Witten appearance of the massless monopole singularity in type IIB. Here we have to remind that study of heterotic string theory, analog of Seiberg-Witten theory, nessessarily involves the calculation of the one loop prepotential and the one loop Kähler metric since effective gauge couplings in $\mathrm{N}=2$ supergravity [56] depend on the moduli of the vector multiplets only. Later, in section five, we will derive the general equation describing the prepotential of $N=2$ compactifications of a heterotic rank three model which has a dual pair, in the sence of [38], described by a IIB model compactified on a Calabi-Yau.

In this work we are interested, among the web of dualities, only in the proposal of [38, 39] which provided evidence for the exact nonperturbative equivalence of dual pairs, the heterotic string compactified on $K_{3} \times T_{2}$, with IIA compactified on a Calabi-Yau threefold. The proposal identifies the moduli spaces of heterotic string and its dual IIA as $\mathcal{M}_{V}^{\text {heterotic }}=\mathcal{M}_{V}^{I I A}$ and $\mathcal{M}_{H}^{\text {heterotic }}=\mathcal{M}_{H}^{I I A}$, where the subscripts refer to vector multiplets and hypermultiplets respectively. In other words, for models which are dual pairs, the exact prepotential for the vector multiplets, including perturbative and non-perturbative corrections is calculated from the IIA side, where the tree level result is exact. On the contrary, if we want to calculate the exact hypermultiplet prepotential this is calculated from the heterotic string on $K_{3} \times T^{2}$. This is exactly Strominger's proposal that the absence of neutral perturbative couplings between vector multiplets and hypermultiplets survives nonperturbative string effects. In this sence the complete prepotentials for the vector multiplets for the two "different" theories match, including perturbative and non-perturbative corrections, and $\mathcal{F}^{\text {het }}=\mathcal{F}^{I I A}$. Compactification of the heterotic string on $K_{3} \times T_{2}$ and 
of type IIA on a Calabi-Yau threefold produce models with $\mathrm{N}=2$ supersymmetry in four dimensions. These models have been tested to be dual to each other, at the level of effective theories, and for low numbers of $h^{(1,1)}$ vector multiplet moduli 38. By using mirror symmetry, we can map the vector multiplet sector of IIA to its mirror type IIB $h^{(2,1)}$ complex structure moduli spacef. The procedure then concentrates in the comparison of the complex structure moduli 96 effective theories, of type IIB, with $N=2$ heterotic compactifications of rank three or four models 38, 98. In this sence, in type IIB the result for the effective gauge coupling $\propto \log (Z)$ is exact to all orders of perturbation theory, since the dilaton in type IIB belongs to the hypermultiplet sector and are no couplings allowed between 116 vector multiplets and hypermultiplets.

The singularity structure of the Calabi-Yau can help us to produce transitions between different vacua by changing the Hodge numbers of the manifold. Note, that for a CalabiYau space exploring the Higgs transitions at the conifold singularities can produce extremal transitions between different Calabi-Yau vacua 42, 43, 44. Exploring singularity transitions in heterotic string compactified in $K_{3} \times T_{2}$, can produce dual models, whose Hodge numbers match known type II models on Calabi-Yau three folds. This was confirmed with the construction of such duals in 66.

In section four, we will calculate the one loop correction to the perturbative prepotential of the vector multiplets for the heterotic string compactified on a six dimensional orbifold. It comes from the general solution from the one loop Kähler metric [57, 10]. The one loop correction to the perturbative prepotential has already been calculated before in [57 from string amplitudes. Our procedure is complementary to [57] since we calculate, contrary to [57 where the third derivative of the prepotential with respect to the $\mathrm{T}$ moduli was calculated, the third derivative of the prepotential with respect to the $\mathrm{U}$ moduli. The logarithmic singularity, consequence of the gauge symmetry enhancement at a specific point in the moduli space do appear in the prepotential and the Kähler metric.

Furthermore, we establish a general procedure for calculating one loop corrections to the one loop prepotential, not only for $N=1$ six dimensional heterotic strings toroidally compactified on four dimensions, which has important implications for any compactification

\footnotetext{
${ }^{6}$ Application of the mirror symmetry on the type IIB interchanges three-cycles with two cycles and three-branes with two branes.
} 
of the heterotic string having(or not) a type II dual. This procedure is an alternative way to the calculation of the prepotential which was performed indirectly in [34] via the effective gauge couplings.

In this work we will continue the work of [10, 56, 57]. We will calculate directly the integral representation of $N=2$ vector multiplet prepotential of toroidal compactifications of the heterotic string of toroidal compactifications of $N=1$ six dimensional orbifold 114 compactifications 62, 80. of the heterotic string follows directly from this result.

In section two we will describe general properties of $N=2$ heterotic strings. In addition, we describe properties of the moduli space of compactification of the heterotic string on a $K_{3} \times T_{2}$ manifold. In addition, we describe elements of the special Kähler geometry describing $N=2$ locally sypersymmetric theory of the heterotic string with emphasis on the couplings of vector multiplets.

In section three we will describe our results for the case of toroidal compactifications of $N=1$ six dimensional orbifold compactification of the heterotic string, where the underlying torus lattice does not "decompose" as $T^{2} \oplus T^{4}$, the $T^{4}$ could be an orbifold limit of $K_{3}$. The moduli of the unrotated complex plane, e.g $T^{2}$ with a shift, has a modular symmetry group that is a subgroup of $S L(2, Z)$. In particular, we consider this modular symmetry, e.g $\Gamma_{0}(3)$, to be one of those appearing in $N=2$ sectors non-decomposable $N=1$ orbifold compactifications [13, 12].

In section four we describe our results for the one loop prepotential of toroidal compactification of the $N=1$ six dimensional heterotic string to four dimensions. In the description of calculating the prepotential of vector multiplets from string amplitudes we will follow the work of [57, 10]. We use string amplitudes to calculate directly the one loop prepotential via its relation to the one loop Kähler metric. The calculation comes from the use of string amplitudes of [10, 57. Automatically this calculates one-loop corrections to the Kähler metric for the moduli of the usual vector multiplet T, U moduli fields of the $T^{2}$ torus appearing in $N=2(4,4)$ compactifications of the heterotic string. The calculation

\footnotetext{
${ }^{7}$ However, due to factorization properties of the $T^{2}$ subspace of the heterotic Narain lattice and instanton embedding independence into the gauge bundle over $K_{3}$, the same result can be applied to any heterotic string compactification on $K_{3} \times T^{2}$ for any rank four models.
} 
on the quantum moduli space takes into consideration points of enhanced gauge symmetry.

In section five we describe our results for the $N=2$ prepotential of any rank three compactifications of the heterotic string. A general result[73] concerning the geometry, local issues, behind the existence of heterotic duals, is that the Calabi-Yau manifold in the IIA side can be written as a fibre bundle with base $8 P^{1}$ and generic fiber the $K_{3}$ surface 69 , while the dual heterotic, on $K_{3} \times T^{2}$, could be written instead as a bundle with base $P^{1}$ and generic fibre $T^{4}$. Existence of the IIA dual in the Calabi-Yau threefold phase, as a global issue, with the dual heterotic string admitting a weekly coupled phase while the dual type IIA realization is in the strongly coupled phase, can happen only when the generic fibre is the $K_{3}$ surface and the base is $P^{1}$. In section five we apply the general procedure for calculating the one loop heterotic prepotential in particular dual type II models compactified on Calabi-Yau, equivalent to rank three heterotic models 20] at a specific limit. The Calabi-Yau models incorporate the $K_{3}$ fiber structure [69] of the type II dual realization.

\section{Properties of $N=2$ heterotic vacua}

\section{Generalities}

In the next section we will calculate the perturbative prepotential using its modular properties for a class of models, possible orbifold limits of $K_{3}$, whose modular group is similar to those appearing $N=2$ sectors of $N=1(2,2)$ symmetric non-decomposable orbifolds. The list of $(2,2)$ vacua includes the Calabi-Yau compactifications [95], orbifolds [17, 18], tensor products of minimal models [4] or generalizations [5]. For abelian $(2,2)$ orbifolds constructed by twisting a six dimensional torus, the point group rotation is accompanied by a similar rotation in the gauge degrees of freedom. The four dimensional gauge group in this case is enlarged beyond $G=E_{6} \otimes E_{8}$ by a factor that can be a $U(1)^{2}, S U(2) \times U(1)$, if $P=Z_{4}$ or $Z_{6}$, or $S U(3)$ if $P=Z_{3}$. If we symbolize by $h^{(1,1)}$ the numberf of $(1,1)$ moduli in the untwisted sector then we have respectively $h^{(1,1)}=3,5$ and

\footnotetext{
${ }^{8} P^{1}$ is the complex projective space with homogeneous coordinates $\left[x_{o}, x_{1}\right]$.

${ }^{9}$ For compactifications on Calabi-Yau manifolds, $h^{(1,1)}$ and $h^{(2,1)}$ represent the Hodge numbers of the manifold.
} 
9. Twisted moduli are not neutral with respect to $\mathrm{G}$ and are not moduli of the orbifold. On the other hand $N=1$ orbifold compactifications of the ten dimensional heterotic string have, in four dimensions, $N=1$ and $N=2$ sectors twisted sectors. The geometry of those $N=2$ sectors is described from special geometry, which will be desribed in the following. Abelian $(2,2)$ orbifolds can flow to a Calabi-Yau vacuum, by blowing up the twisted moduli fields, giving them vacuum expectation values[6]. The $N=2$ sectors exhibit modular groups which are similar to those appearing to $N=2$ supersymmetric Yang-Mills with number of hypermultiplets zero or two 81, 82.

Of particular interest in this work is the $N=2$ toroidal compactifications of the six dimensional heterotic vacua. The moduli of the two torus is parametrized from the relations $T=2(B+i \sqrt{G})$ and $U=1 / G_{11}\left(G_{12}+\sqrt{G}\right)$, where $G_{i j}$ is the lattice metric of the $T^{2}, \sqrt{G}$ is determinant and $\mathrm{B}$ the constant antisymmetric tensor background field. The moduli T, $\mathrm{U}$ represent the scalar components of two $N=2$ vector multiplet fields in four dimensions.

At the classical level, moduli are described by a flat potential [3, 28, 27] to all orders of perturbation theory. Compactifications of the heterotic string in four dimensions with $N=2$ supersymmetry involve a $U(1) \times U(1)$ gauge group from the untwisted $T^{2}$ subspace. The T, U moduli subspace, exhibits an $S L(2, Z)_{T} \times S L(2, Z)_{U} \times Z_{2}^{T \leftrightarrow U}$ classical duality group, and corresponds to the coset space $\left.\frac{S O(2,2)}{S O(2) \times S O(2)}\right|_{T, U}$. The same type of moduli appears when we further compactify 62, 34, the $N=1$ six dimensional heterotic string compactified on the manifold $K_{3}$, on a two torus. The gauge group associated with the two torus can be futher enhanced at special points in the moduli space, namely the $T=U$ line the gauge group becomes enhanced to $S U(2) \times U(1)$. It can become enhanced to $S O(4)$ or $S U(3)$ along the $T=U=i$ or $T=U=e^{2 \pi i / 3}$ lines respectively. In general the classical moduli space, of $\mathrm{r}$ vector multiplets in the $T^{2}$ subspace, is the group 111

$$
\left.\frac{S U(1,1)}{U(1)}\right|_{\text {dilaton }} \times \frac{O(2, r)}{O(2) \times O(r)} / O(2, r ; Z),
$$

where the first factor corresponds to the complex dilaton $\mathrm{D}$

S. For our case, the classical duality group comes with $r=2$. Here, $O(2,2 ; Z)$ is the target space duality group. The theory enjoy the non-trivial global invariance i.e identifications under target space duality symmetries [101, [] the $P S L(2, \mathbf{Z})_{T} \times P S L(2, \mathbf{Z})_{U}$ 
dualities acting as

$$
T \rightarrow \frac{a T-i b}{i c T+d}, \quad U \rightarrow \frac{a^{\prime} U-i b^{\prime}}{i c^{\prime} U+d^{\prime}}
$$

The same vector multiplet, as eqn.(2.1) appears for generic $(4,4)$ compactifications of the heterotic string on the $K_{3} \times T^{2}$. For compactifications on $K_{3}$ manifolds, the moduli space of metrics with $S U(2)$ holonomy associated to complex and Kähler deformations is $\mathcal{M}=\frac{S O(19,3)}{S O(19) \times S O(3) \times S O(19,3 ; Z)} \times R^{+}$, where $R^{+}$is associated 48 with the volume of $K_{3}$. Adding the moduli coming from deformations of the antisymmetric tensor we get the moduli space of hypermultiplets[105] of $K_{3} \frac{S O(20,4)}{S O(20) \times S O(4,20 ; Z) \times S O(4)}$.

Lets us now describe some properties $\$$ of the low energy effective actions of $N=2$ effective string theories. In $N=2$ supersymmetric Yang-Mills theory the action for the vector multiplets is described by a holomorphic prepotential $\tilde{F}(X)$, where $X^{A}(A=1, \ldots, n)$ are the complex scalar components of the corresponding vector superfields. The couplings of the classical vector multiplets with supergravity are determined by another holomorphic function $F(X)$, the prepotential function which is a holomorphic function of $n+1$ complex variables $X^{I}(I=0,1, \ldots, n)$ and it is a homogeneous function of degree two 107 in the fields $X^{I}$. However, in $N=2$ supergravity theories, supersymmetry demands an additional vector superfield $X^{0}$ which account for the accommodation of the graviphoton. It stands for the $I=0$ component of the vector multiplets and it belongs to a compensating multiplet. The graviphoton is the vector component of the compensating multiplet and is the spin one gauge boson of the $N=2$ supergravity multiplet. The coordinate space of physical scalar fields belonging to vector multiplets of an $N=2$ supergravity [107, 109] is described from special Kähler geometry [111], with the Kähler metric $g_{A B}=\partial_{A} \partial_{\bar{B}} K(z, \bar{z})$ resulting from a Kähler potential of the form

$$
K(z, \bar{z})=-\log \left(i \overline { X } ^ { I } ( \overline { z } ) F _ { I } \left(X(z)-i X^{I}(z) \bar{F}_{I}(\bar{X}(\bar{z})), F_{I}=\frac{\partial F}{\partial X_{I}}, \bar{F}_{I}=\frac{\partial F}{\partial \bar{X}_{I}} .\right.\right.
$$

The spectrum of $N=2$ heterotic strings in four dimensions contains, among other fields, the dilaton $\mathrm{D}$ and the antisymmetric tensor $\square$ and the graviton. The axion is subject to the discrete Peccei-Quinn symmetry to all orders of perturbation theory. Since the axion is connected through a duality transformation to the antisymmetric tensor field, whose vertex

\footnotetext{
${ }^{10}$ For a review of $N=2$ heterotic strings see 56 .

${ }^{11}$ connected to the axion via a duality transformation
} 
operator decouples at zero momentum, this means that every physical amplitude involving $B_{\mu \nu}$ at zero momentum is zero. As a result the effective theory of the heterotic superstring is independent of the field $B_{\mu \nu}$ at zero momentum and the coupling of field appear only through its derivative. The dilaton and the axion belong to a vector multiplet. Since the axion couples to the dilaton $\mathrm{D}$ via the complex scalar $\mathrm{S}$, which we will refer next as the dilaton, we conclude that any dependence of holomorphic quantities, e.g the Wilsonian gauge couplings, will be through the combination $S+\bar{S}$. However, these arguments are not valid non-perturbatively. As a result the non-perturbative corrections to the prepotential of $N=2$ compactifications of the heterotic string depend on S. However, they will be of no particular interest to us, since we will discuss the one loop correction to the prepotential at the semiclassical limt $S \rightarrow \infty$.

The prepotential for the classical moduli space of vector multiplets reads

$$
F(X)=-\frac{X^{1}}{X^{0}}\left[X^{2} X^{3}-\sum_{I=4}^{n}\left(X^{I}\right)^{2}\right]
$$

while the values of the moduli are identified as

$$
S=-i \frac{X^{1}}{X^{0}}, \quad T=-i \frac{X^{2}}{X^{0}}, \quad U=-i \frac{X^{3}}{X^{0}}, \quad \phi^{i}=-i \frac{X^{i+3}}{X^{0}}, \quad(i=1, \ldots, P)
$$

with the remaining $X^{I}, C^{a}=-i X^{a+P+3} / X^{0}, a=p+4, \ldots, n$ correspond [56, 111] to matter scalars. Matter scalars are members of the hypermultiplets while the scalars in the Cartran subalgebra of the non-abelian gauge group of the heterotic vacuum can be used to break the gauge group.

From the values of the moduli previously given, it follows that the the Kähler potential is

$$
K=-\log \left((S+\bar{S})\left[(T+\bar{T})(U+\bar{U})-\sum_{i}\left(\phi^{i}+\bar{\phi}^{i}\right)^{2}-\sum_{a}\left(C^{a}+\bar{C}^{a}\right)^{2}\right)\right.
$$

\section{One loop prepotential - perturbative aspects}

Since we have finish our discussion of the general properties of the $N=2$ heterotic strings, we will now discuss the calculation of perturbative corrections to the one loop prepotential. We are interested on those heterotic strings which exhibit modular groups simi- 
lar to those appearing in the calculation of thresholds corrections in non-decomposable[13] $N=1$ symmetric orbifolds 12$]$.

Let us expand at the moment the expression of eqn.(2.4) around small values of the non moduli scalars $C_{a}$ as in (2.6)

$$
F=-S\left(T U-\sum_{i} \phi^{i} \phi^{i}\right)+h\left(T, U, \phi^{i}\right)
$$

or

$$
\begin{array}{r}
F=-d_{s i j} T^{i} T^{j} S+h\left(T, U, \phi^{i}\right), \quad d_{s i j}=\operatorname{diag}(+,-, \ldots,-) \\
T^{1}=T, T^{2}=U, T^{i}=\phi^{i}, i \neq 1,2 .
\end{array}
$$

The function $h$, the one loop correction to the perturbative prepotential, enjoy a nonrenormalization theorem, namely it receives perturbative corrections only up to one loop order. Its higher loop corrections, in terms of the $1 /(S+\bar{S})$, vanish due to the surviving of the discrete Peccei-Quinn symmetry to all orders of perturbation theory as a quantum symmetry.

In that case, under target space duality

$$
T \stackrel{S L(2, Z)_{T}}{\rightarrow} \frac{a T-i b}{i c T+d}, \quad U \stackrel{S L(2, Z)_{T}}{\rightarrow} U
$$

we get

$$
h(T, U) \stackrel{S L(2, Z)_{T}}{\rightarrow} \frac{h(T, U)+\Xi(T, U)}{(i c T+d)^{2}}
$$

and a similar set of transformations under $P S L(2, Z)_{U}$. The net result is that $\partial_{T}^{3} h^{(1)}(T, U)$ is a singled valued function of weight -2 under U-duality and 4 under T-duality. The prepotential h modifies the Kähler potential, in the lowest order of expansion in the matter fields, as

$$
K=-\log \left[(S+\bar{S})+V_{G S}\right]-\log (T+\bar{T})^{2}-\log (U+\bar{U})^{2}
$$

where

$$
V_{G S}=\frac{2(h+\bar{h})-(T+\bar{T})\left(\partial_{T} h+\partial_{\bar{T}} \bar{h}\right)-(U+\bar{U})\left(\partial_{U} h+\partial_{\bar{U}} \bar{h}\right)}{(T+\bar{T})(U+\bar{U})}
$$

is the Green-Schwarz term 108] which contains the mixing of the dilaton with the moduli

${ }^{12}$ In the next section, we will see practically the direct calculation of the $V_{G S}$ via the calculation of h. However, our notation will be different following the spirit of [57] and $\mathrm{h}$ will be denoted by $\mathrm{f}$. 
at one loop order. Rememebr, that for the conventions used at this section the dilaton is defined as $<S>=4 \pi / g^{2}-i \theta / 2 \pi$.

The prepotential for $N=2$ orbifold compactifications of the heterotic string 62, 197, 13]. was calculated, from the use of its modular properties and singularity structure in [56]. Here, we adopt a similar approach to calculate the prepotential of vector multiplets. We discuss the calculation of the prepotential for the case where the moduli subspace of the Narain lattice associated with the T, U moduli exhibits a modular symmetry 12, 29, 30 group $\Gamma^{o}(3)_{T} \times \Gamma^{o}(3)_{U}$. The same modular symmetry group appears 12 in the $N=2$ sector of the $N=1(2,2)$ symmetric non-decomposable $Z_{6}$ orbifold defined on the lattice $S U(3) \times S O(8)$. In the third complex plane associated with the square of the complex twist $(2,1,-3) / 6$ the mass operator for the untwisted subspace was given $\$$ [?

$$
m^{2}=\sum_{m_{1}, m_{2}, n_{1}, n_{2} \in Z} \frac{1}{2 T_{2} U_{2}}\left|T U^{\prime} n_{2}+T n_{1}-U^{\prime} m_{1}+3 m_{2}\right|^{2}, \quad U^{\prime}=U+2 .
$$

Let us forget the $N=1$ orbifold nature of the appearance of this $N=2$ sector. Then its low energy supergravity theory is described by the underlying special geometry. The question now is if calculating the prepotential using its modular properties and the singularity structure, as this was calculated for decomposable orbifold compactifications of the heterotic string[56], has any type II dual realization. We believe that it is the case. In the analysis of the map between type II and heterotic dual supersymmetric string theories [96, 69] it was shown that subgroups of the modular group do appear. In particular some type II compactified on the Calabi-Yau three folds[38], were shown 69] to correspond in one modulus deformations of $K_{3}$ fibrations. The modular symmetry groups appearing 96 are all connected to the $\Gamma_{o}(N)_{+}$, the subgroup of the $P S L(2, Z)$, the $\Gamma_{o}(N)$ group together with the Atkin-lehner involutions $T \rightarrow \frac{-1}{N T}$. We expect that the same prepotential, beyond describing the geometry of the $N=2$ sector of $Z_{6}$ in exact analogy to the decomposable case, may come form a compactification of the heterotic string on the $K_{3} \times T^{2}$. An argument that seems to give some support to our conjecture was given in [73]. It was noted by Vafa and Witten that if we compactify a ten dimensional string theory on $T^{2} \times X$, where $\mathrm{X}$ any

\footnotetext{
${ }^{13}$ We changed notation. All moduli are rescaled by i.

${ }^{14}$ We use this term in connection with the same type of modular symmetries appearing in $N=1$ decomposable orbifold compactifications of the heterotic string 17 .
} 
four manifold, acting with a $Z_{2}$ shift on the Narain lattice we get the modular symmetry group $\Gamma_{o}(2)_{T} \times \Gamma_{o}(2)_{U}$. In this respect it is obvious that our calculation of the prepotential may come from a shift in a certain Narain lattice of $T^{2}$. We suspect that this is a $Z_{3}$ shift. Furthermore, if we adopt $\mathrm{N}=2$ conventions [81] in the study of dyon spectrum of $N=2 \mathrm{su}-$ persymmetric Yang-Mills, the quantum symmetry groups $\Gamma_{o}(2), \Gamma^{o}(2)$ appear in $N_{f}=0,2$ respectively with corresponding monodromy groups $\Gamma^{o}(4), \Gamma_{o}(4)$.

From the mass operator (3.7) we deduce that at the point $T=U$ in the moduli space of the $T^{2}$ torus of the untwisted plane, with $n_{1}=m_{1}= \pm 1$ and $n_{2}=m_{2}=0$, its $U(1) \times U(1)$ symmetry becomes enhanced to $S U(2) \times U(1)$. Moreover, the third derivative of the prepotential has to transform, in analogy to the $S L(2, Z)$ case, with modular weights -2 under $\Gamma^{o}(3)_{U}$ and 4 under $\Gamma^{o}(3)_{T}$ dualities. Using the theory of modular forms requires, for the calculation of the vector multiplet prepotential of the effective $\mathrm{N}=2$ low energy theory of heterotic strings, the analog of $S L(2, Z)$ j-invariant, for $\Gamma^{o}(3)$, the hauptmodul function. This quantity is given by $\omega(T)$, where $\omega(T)$ is given explicitly by

$$
\omega(T)=\left(\frac{\eta(T / 3)}{\eta(T)}\right)^{12}
$$

and represents the hauptmodul for $\Gamma^{0}(3)$, the analogue of $j$ invariant for $S L(2, \mathbf{Z})$. It is obviously automorphic under $\Gamma^{0}(3)$ and possess 5 a double pole at infinity and a double zero at zero. It is holomorphic 115 in the upper complex plane and at the points zero and infinity has the expansions

$$
\omega(T)=t_{\infty}^{-1} \sum_{\lambda=0}^{\infty} a_{\lambda} t_{\infty}^{\lambda}, a_{o} \neq 0, \omega(T)=t_{o}^{-1} \sum_{\lambda=0}^{\infty} b_{\lambda} t_{o}^{\lambda}, b_{o} \neq 0
$$

at $\infty$ and 0 respectively with $t=e^{-2 \pi T}$.

In full generality, the hauptmodul functions for the $\Gamma^{0}(p)$ are the functions 24, 26]

$$
\Phi(\tau)=\left(\frac{\eta\left(\frac{\tau}{p}\right)}{\eta(\tau)}\right)^{r} .
$$

Here, $p=2,3,5,7$ or 13 and $r=24 /(p-1)$. For these values of $\mathrm{p}$ the function in eqn.(3.10) remains modular invariant, i.e it is a modular function. The hauptmodul functions for the group $\Gamma_{0}(p)$ are represented by

$$
\left(\frac{\eta(\tau)}{\eta(p \tau)}\right)^{r} .
$$

\footnotetext{
${ }^{15}$ We would lile to thank D. Zagier for pointing this to us.
} 
The function $\omega^{o}(3)$ has a single zero at zero and a single pole at infinity. In addition, its first derivative has a first order zero at zero, a pole at infinity and a first order zero at $i \sqrt{3}$. The modular form $\mathrm{F}$ of weight $\mathrm{k}$ of a given subgroup of the modular group $P S L(2, Z)=S L(2, Z) / Z_{2}$ is calculated from the formula

$$
\sum_{p \neq 0, \infty} \nu_{p}+\sum_{p=0, \infty}(\text { width }) \times(\text { order of the point })=\frac{\mu k}{12} .
$$

Here, $\nu_{p}$ the order of the function $\mathrm{F}$, the lowest power in the Laurent expansion of $\mathrm{F}$ at $\mathrm{p}$. The index $\mu$ for $\Gamma^{o}(3)$ is calculated from the expression 115

$$
\left[\Gamma: \Gamma_{o}(N)\right]=N \Pi_{p / N}\left(1+p^{-1}\right)
$$

equal to four. The width at infinity is defined as the smallest integer such as the transformation $z \rightarrow(z+\alpha)$ is in the group, where $\alpha \in Z$. The width at zero is coming by properly transforming the width at infinity at zero. For $\Gamma^{o}(3)$ the width at infinity is 3 and the width at zero is 1 . The holomorphic prepotential can be calculated easily if we examine its seventh derivative. The seventh derivative has modular weight 12 in $\mathrm{T}$ and 4 in U. In addition, it has a sixth order pole at the $T=U$ point whose coefficient A has to be fixed in order to produce the logarithmic singularity of the one loop prepotential. As it was shown[57, 56] the one loop prepotential as $\mathrm{T}$ approaches $U_{g}=\frac{a U+b}{c U+d}$, where $\mathrm{g}$ is an $S L(2, Z)$ element四

$$
f \propto-\frac{i}{\pi}\{(c U+d) T-(a U+b)\}^{2} \ln \left(T-U_{g}\right) .
$$

The seventh derivative of the prepotential is calculated to be

$$
f_{\text {TTTTTTT }}=A \frac{\omega(U)_{U}^{3} \omega(U)^{5}\left(\omega^{\prime}(U)\right)^{3}}{(\omega(U)-\omega(\sqrt{3}))^{2}(\omega(U)-\omega(T))^{6}} X(T),
$$

where $X(T)$ a meromorphic modular form with modular weight 12 in $\mathrm{T}$. The complete form of the prepotential is

$$
f_{\text {TTTTTTT }}=A\left(\frac{\left[\omega(U)_{U}^{3} \omega(U)^{5}\left(\omega^{\prime}(U)\right)^{3}\right.}{(\omega(U)-\omega(\sqrt{3}))^{2}\left[(\omega(U)-\omega(T))^{6}\right]}\right)\left(\frac{\omega(T)_{T}^{6}}{\omega^{2}(T)\left\{(\omega(T)-\omega(\sqrt{3}))^{4}\right\}}\right) .
$$

\footnotetext{
${ }^{16}$ The same argument works for the subgroups of the modular group, but now there are additional restrictions on the parameters of the modular transformations.
} 
The two groups $\Gamma^{o}(3)$ and $\Gamma_{o}(3)$ are conjugate to each other. If $\mathrm{S}$ is the generator

$$
S=\left(\begin{array}{cc}
0 & -1 \\
1 & 0
\end{array}\right), \text { we have } \Gamma^{o}(3)=S^{-1} \Gamma_{o}(3) S
$$

So any statement about modular functions on one group is a statement about the other. We have just to replace everywhere $\omega(z)$ by $\omega(3 z)$ to go from a modular function from the $\Gamma^{o}(3)$ to the $\Gamma_{o}(3)$. In other words, the results for the heterotic prepotential with modular symmetry group $\Gamma^{o}(3)$ may well be describe the prepotential to the conjugate modular theory.

We have calculated the prepotential of a heterotic string with a $\Gamma^{o}(3)_{T} \times \Gamma^{o}(3)_{T} \times$ $Z_{2}^{T \leftrightarrow U}$ classical duality group. The same dependence on the $\mathrm{T}, \mathrm{U}$ moduli and its modular symmetry group appears in the $\Theta^{2}, N=2$, sector of the $Z_{6}$ orbifold defined by the action of the complex twist $\Theta=\exp \left[\frac{2 \pi i}{6}(2,3,-1)\right]$ on the six dimensional lattice $S U(3) \times S O(8)$, namely the $Z_{6}$-IIb.

If our weakly coupled heterotic model has a non-perturbatively equivalent Calabi-Yau dual type IIA model then it has to come from generic fibers of a $K_{3}$ fibration. The generic fibers can be seen from the non-zero entries of the intersection numbers $\square$

$$
\left(D_{s i j}\right)=\operatorname{diag}(+,-, \ldots,-, 0, \ldots, 0)
$$

The zero entries correspond to singular fibers, fibers which degenerate at points in the moduli space, to non $K_{3}$ surfaces like a smooth manifold, and correspond to the heterotic side to strong coupling singularities [94, 25] seen non-perturbatively 67. Because of the maximum number of $K_{3}$ moduli 20, the number of generic fibers is constrained to be less than 20. We believe that the nature of the lattice twist of non-decomposable orbifolds is such that its form when acting on the $N=2$ planes may correspond to orbifold limits $K_{3}$. In this phase, the $K_{3}$ surface can be written as an orbifold of $T^{4}$. The fixed points of $T^{4}$ under the orbifold action are the singular limits of $K_{3}$ because the metric on the fixed point develops singularities. The singularities of $K_{3}$ follow an ADE classification pattern. In fact, because at the adiabatic limit 73 , we can do even the reverse, we can map the type

\footnotetext{
${ }^{17}$ See for example (5.4) and for more details section five.

18 C. Kounnas has suggested to me that some of them might correspond to freely acting orbifolds.
} 
II phase to the heterotic one. In the limit where the base of the fibration has a large area, but the volume of the $K_{3}$ fiber is of order one, we can replace the $K_{3}$ fibers in the heterotic side with $T^{4}$ fibers over a $P^{1}$ base. In this form, the heterotic string description is replaced by $T^{4}$ fibers, namely the Narain lattice $\Gamma^{20,4}$.

\section{One loop correction to the prepotential from string ampli- tudes}

In the section (4.1) we review general properties involved in the calculation of the one loop Kähler metric and the one loop prepotential f. Then we discuss properties of the equation for $f_{U U U}$ and finally we introduce the equation for the rank four $N=2$ heterotic string compactifications.

\subsection{One loop contribution to the Prepotential/ Kähler metric}

Preliminaries - Rank four models

The one-loop Kähler metric for orbifold compactifications of the heterotic string, where the internal six torus decomposes into $T^{2} \oplus T^{4}$, was calculated in [10. In this section, we will use the general form of the solution for the one loop Kähler metric appearing in [10, 57] to calculate the one loop correction to the prepotential of $N=2$ orbifold compactifications of the heterotic string. While the one loop prepotential has been calculated with the use of string amplitudes in [57) via its third derivative with respect to the $\mathrm{T}$ moduli, here we will provide an alternative way of calculating the one-loop correction 56, 57 to the prepotential of the vector multiplets of the $N=2$ orbifold compactifications of the heterotic string. For the calculation of the one-loop contribution to the Kähler metric we use the linear multiplet formulation[102, 10] and not the chiral formulation. Note that both formulations are equivalent, since the linear multiplet can always be transformed in to a chiral multiplet by a supersymmetric duality transformation.

In the superconformal formalism[103, the action for the linear multiplet is given up to 
one loop order by

$$
\mathcal{L}=-\left(S_{o} \bar{S}_{o}\right)^{3 / 2}\left(\frac{\hat{L}}{2}\right)^{-\frac{1}{2}} e^{-\frac{G^{(o)}}{2}}+\left(\frac{\hat{L}}{2}\right) G^{(1)}+\left(S_{o}^{3} w\right)_{F}
$$

where now the gauge kinetic function is given by $G^{(o)}(z, \bar{z})+l G^{(1)}(z, \bar{z})$. The vev of $l$ is the four dimensional gauge coupling constant $g^{2}$. Here, $\hat{L}$ is the real linear multiplet satisfying $\mathcal{D}^{2} \hat{L}=0$.

Eqn.(4.1) does not have the the gravitational kinetic energy $\propto R$ term to its canonical form. Instead, the chiral compensator field is used to properly normalising its coefficient, procedure which fixes the value of the compensator field. The advantage of using the linear multiplet instead of the chiral multiplet is that it provides an easy way of calculating 10 one loop corrections to the Kähler metric. An easy way to see this comes from the following equation 5 , which includes the bosonic kinetic energy terms,

$$
\mathcal{L}_{\text {bosonic }}=-\frac{1}{4 l^{2}} \partial_{\mu} l \partial^{\mu} l+\frac{1}{4 l^{2}} h^{\mu} h_{\mu}-G_{i \bar{j}} \partial_{\mu} z^{i} \partial^{\mu} \bar{z}^{\bar{j}}-\frac{i}{2}\left(G_{i j} \partial z^{j}-G_{i \bar{j}} \partial_{\mu} z^{j}-G_{i \bar{j}} \partial_{\mu} \bar{z}^{\bar{j}}\right) h^{\mu}
$$

The last term in eqn.(4.2) reveals that the one loop correction to the Kähler metric $G_{z, \bar{z}}$ will come by calculating the CP-odd part of the amplitude between the complex scalars and the antisymmetric tensor $b^{\mu \nu}$

$$
<z\left(p_{1}\right) \bar{z}\left(p_{2}\right) b^{\mu \nu}\left(p_{3}\right)>_{o d d}=i \epsilon^{\mu \nu \lambda \rho} p_{1 \lambda} p_{2 \rho} G_{z \bar{z}}^{(1)}
$$

Here, $\mathrm{G}$ is the Kähler metric and $h^{\mu}=\frac{1}{2} \epsilon^{\mu \nu \lambda \rho} \partial_{\nu} b_{\lambda \rho}$ is the dual field strength of the antisymmetric tensor field $b_{\lambda \rho}$.

The amplitude receives contributions only from $N=2$ sectors. We are not considering contributions to the Kähler metric which arise from $N=1$ sectors, since these contributions arise only in $N=1$ orbifold compactifications of the heterotic string. Here, we are only interested in the geometry underlying the $N=2$ sectors. Remember, than in general string amplitudes involve not only diagrams that can be characterized 1PI diagrams in comparison with the corresponding effective field theory diagrams. Rather, they include one-particle reducible ones. Integrating out heavy fields in effective field theories leaves terms associated with non-renormalizable interactions. In string theory, integration of

\footnotetext{
${ }^{19}$ Coming by expanding eqn.(4.1).
} 
massive states produces holomorphic quantities in the effective string action. The presence of additional massive fields becoming massless in a region of moduli space, produces nonholomorphic quantities, e.g a logarithmic singularity at weak Wilsonian gauge coupling [19].

Lets us suppose that the internal six dimensional lattice decomposes into $T^{2} \oplus T^{4}$. The $T^{4}$ part of the lattice, may represent an orbifold limit 97, 62] of $K_{3}$ while the $T^{2}$ part, which contains the usual T, U moduli may contain 20 a lattice shift, necessary for modular invariance. In this subspace of the Narain moduli space, we want to calculate the moduli dependence of the one loop correction to the prepotential. Denote the untwisted moduli from a $N=2$ sector by $\mathrm{P}$, where $\mathrm{P}$ can be the $\mathrm{T}$ or $\mathrm{U}$ moduli parametrizing $[7]$ the two dimensional unrotated plane. Then the one loop contribution 10 to the Kähler metric is given by

$$
G_{P \bar{P}}^{(1)}=-\frac{1}{(P-\bar{P})^{2}} \mathcal{I}, \quad \mathcal{I}=\int_{\mathcal{F}} \frac{d^{2} \tau}{\tau_{2}^{2}} \partial_{\bar{\tau}}\left(\tau_{2} Z\right) \bar{F}(\bar{\tau}) .
$$

Here, the integral is over the fundamental domain, and the factor $-\frac{1}{(P-\bar{P})^{2}}$ is the tree level moduli metric $G_{P \bar{P}}^{(0)}$. Z is the partition function of the fixed torus

$$
Z=\sum_{\left(P_{L}, P_{R}\right) \in \Gamma_{2,2}} q^{P_{L}^{2} / 2} \bar{q}_{R}^{2} / 2, q \equiv e^{2 \pi \tau} \tau=\tau_{1}+\tau_{2}
$$

and $P_{L}, P_{R}$ are the left and right moving momenta associated with this plane. $F(\tau)$ is a moduli independent meromorphic form² of weight -2 with a single pole at infinity due to the tachyon at the bosonic sector. The function F was fixed in [57] to be

$$
F(\tau)=-(1 / \pi) \frac{j(\tau)[j(\tau)-j(i)]}{j_{\tau}(\tau)}, \quad j_{\tau} \stackrel{\operatorname{def}}{=} \frac{\partial j(\tau)}{\partial \tau}
$$

where $\mathrm{j}$ the modular function for the group $S L(2, Z)$. The function $F(\tau)$ is actually the index in the Ramond sector in the in the remaining superconformal blocks. With the use of the relations between $E_{4}, E_{6}, \triangle$ and $\mathrm{j}$, appearing in the Appendix, we can easily see that $\mathrm{F}$ becomes

$$
F(\tau)=-2 i \frac{1}{(2 \pi)^{2}} \frac{E_{4}(\tau) E_{6}(\tau)}{\triangle(\tau)}
$$

\footnotetext{
${ }^{20}$ As it happens in $N=2(4,0)$ models 57,62 .

${ }^{21} \mathrm{~A}$ function $\mathrm{f}$ is meromorphic at a point $\mathrm{A}$ if the function $\mathrm{h}, h(z) \stackrel{\text { def }}{=}(z-A) f(z)$ is holomorphic (differentiable) at the point A. In general, this means that the function $\mathrm{h}$ is allowed to have poles.
} 


\section{Prepotential of vector multiplets/Kähler metric}

The convention for the complex dilaton is $\langle S\rangle=\frac{\theta}{\pi}+i \frac{8 \pi}{g_{s}^{2}}$, where $g_{s}$ is the four dimensional string coupling and the $\theta$ angle. For the calculation of the prepotential of the vector multiplets we will will follow the approach of [57]. Recalling the general form of the Kähler potential

$$
\begin{gathered}
K=-\ln (i Y), Y=2(F-\bar{F})-(T-\bar{T})\left(\left(F_{T}+\bar{F}_{\bar{T}}\right)-(S-\bar{S})\left(F_{S}+\bar{F}_{\bar{S}}\right),\right. \\
F=S T U+f(T, U)+f^{n o n-p e r t} .
\end{gathered}
$$

In the following we will neglect the non-perturbative contributions $f^{N P}$ to f. The lagrangian (4.1) may be related to the chiral multiplet one, by a duality transformation. We introduce the dilaton $\mathrm{S}$ as a Lagrange multiplier into 4.1), e.g $(\mathcal{L}-\operatorname{Li}(S-\bar{S}) / 4)_{D}$. Using the equation of motion for $\mathrm{S}$ we get

$$
(\mathcal{L}-L \partial \mathcal{L})_{D} \equiv \frac{-3}{2} S_{o} \bar{S}_{o} e^{-\frac{K}{3}}
$$

In this form the Kähler potential has an expansion as

$$
K=-\ln \left\{i(S-\bar{S})-2 i G^{(1)}\right\}+G^{(o)}
$$

The quantity $G^{(1)}$ is identified with the Green-Schwarz term at the semiclassical weak coupling limit $S \rightarrow \infty$. Expanding (4.11)

$$
K_{P \bar{P}}^{(1)}=\frac{2 i}{(S-\bar{S})} G_{P \bar{P}}^{(1)}, \quad G_{T \bar{T}}^{(1)}=\frac{i}{2(T-\bar{T})^{2}}\left(\partial_{T}-\frac{2}{T-\bar{T}}\right)\left(\partial_{U}-\frac{2}{U-\bar{U}}\right) f+c . c .
$$

Using the equations for the momenta

$p_{L}=\frac{1}{\sqrt{2 \operatorname{ImTImU}}}\left(m_{1}+m_{2} \bar{U}+n_{1} \bar{T}+n_{2} \bar{U} \bar{T}\right), p_{R}=\frac{1}{\sqrt{2 \operatorname{ImTImU}}}\left(m_{1}+m_{2} \bar{U}+n_{1} T+n_{2} T \bar{U}\right)$,

we can prove that $\mathcal{I}$ satisfies 10 the following differential equation

$$
\left\{\partial_{T} \partial_{\bar{T}}+\frac{2}{(T-\bar{T})^{2}}\right\} \mathcal{I}=-\frac{4}{(T-\bar{T})^{2}} \int d^{2} \tau \bar{F}(\bar{\tau}) \partial_{\tau}\left(\partial_{\bar{\tau}}^{2}+\frac{i}{\tau_{2}} \partial_{\bar{\tau}}\right)\left(\tau_{2} \sum_{P_{L}, P_{R}} q^{P_{L}^{2} / 2} \bar{q}^{P_{R}^{2} / 2}\right) .
$$

The integral representation of eqn.(4.14) is a total derivative with respect to $\tau$ and thus zero. However, the integral can give non-vanishing contributions at the enhanced symmetry points $T=U$. 
The general solution of (4.14) away of the enhanced symmetry points is 57

$$
\mathcal{I}=\frac{1}{2 i}\left(\partial_{T}-\frac{2}{T-\bar{T}}\right)\left(\partial_{U}-\frac{2}{U-\bar{U}}\right) f(T, U)+c . c
$$

Note that $\mathrm{f}$ represents the one-loop correction to the prepotential of the vector multiplets $\mathrm{T}$, $\mathrm{U}$ and determines via eqn.(4.12) the one loop correction to the Kähler metric for the T, U moduli. While $f_{T T T}$ was calculated for $N=2$ compactifications of the heterotic string in $D=4$ through its modular properties [56] and from string amplitudes [57, f could only be calculated indirectly [34]. Up to now the only way of calculating $\mathrm{f}$ for heterotic string compactifications was indirectly [34], through the one loop corrections to the Wilsonian gauge couplings [21, 22, 11] in the form

$$
\partial_{U} \partial_{\bar{U}} \triangle=b K_{U \bar{U}}^{(o)}+4 \pi^{2} K_{U \bar{U}}^{(1)}
$$

Here, $\triangle$ is given by $87,88,89,16$

$$
\triangle=-\frac{1}{2} \int_{\mathcal{F}} \frac{d^{2} \tau}{\tau_{2}} \frac{1}{\eta^{2}(\bar{\tau})} \operatorname{Tr}_{R} F(-)^{F}\left(T_{a}^{2}-\frac{1}{2 \pi \tau_{2}}\right)
$$

where the trace is over the R-R sector, $T_{a}$ is the gauge group generator of the gauge group factor $G_{a}, \mathrm{~F}$ is the worldsheet fermion number. In addition, $K_{U \bar{U}}^{(o)}$ is the tree level Kähler metric [10, 34] $-1 /(U-\bar{U})^{2}$ and $K_{U U}^{(1)}$ is the one loop Kähler metric given by

$$
K_{U \bar{U}}^{(1)}=-\frac{1}{8 \pi^{2}} \int_{\mathcal{F}} \frac{d^{2} \tau}{\tau_{2}} \partial_{U} \partial_{\bar{U}}\left(\sum_{B P S \text { hypermultiplets }}-\sum_{B P S \text { vector multiplets }}\right) e^{i \pi \tau M_{L}^{2}} e^{-i \pi \bar{\tau} M_{R}^{2}},
$$

where $M_{L}, M_{R}$ are the masses for the left and right movers and $\triangle$ is defined in terms of gauge couplings as

$$
\frac{4 \pi^{2}}{g^{2}}=\frac{\pi}{2} \operatorname{Im} S+\triangle
$$

In [57] it was shown function $f(T, U)$ of (4.15) satisfies the differential equation

$$
-i(U-\bar{U}) D_{T} \partial_{T} \partial_{\bar{U}} \mathcal{I}=\partial_{T}^{3} f
$$

where $D_{T}=\partial_{T}+\frac{2}{(T-\bar{T})}$ is the covariant derivative. Expansion of the l.h.s and integration by parts results in

$$
f_{T T T}=4 \pi^{2} \frac{U-\bar{U}}{(T-\bar{T})^{2}} \int d^{2} \tau \bar{F}(\bar{\tau}) \sum_{P_{L}, P_{R}} P_{L} \bar{P}_{R}^{3} q^{P_{L}^{2} / 2} \bar{q}_{R}^{2} / 2
$$


Examination of the behaviour of the r.h.s of eqn.(4.21) under separately modular transformations $S L(2, Z)_{T}, S L(2, Z)_{U}$, together with examination of its singularity structure at the enhanced symmetry point $T=U$, uniquely determines the well known solution of the third derivative of the vector multiplet prepotential. Remember that we examine the behaviour of the prepotential including the region of the moduli space where we have gauge symmetry enhancement to $U(1) \times S U(2)$.

For $N=2$ heterotic strings compactified on decomposable orbifolds $f_{T T T}$ was found to be 57

$$
f_{T T T}=-\frac{2 i}{\pi} \frac{j_{T}(T)}{j(T)-j(U)}\left\{\frac{j(U)}{j(T)}\right\}\left\{\frac{j_{T}(T)}{j_{U}(U)}\right\}\left\{\frac{j(U)-j(i)}{j(T)-j(i)}\right\} .
$$

In [56, 57 $f_{T T T}$ was determined by the property of behaving as a meromorphic modular form of weight 4 under T-duality. In addition, $f_{T T T}$ had to vanish at the order 2 fixed point $U=i$ and the order 3 fixed point $U=\rho$ of the modular group $S L(2, Z)$. Moreover, it had to transform with modular weight -2 under $S L(2, Z)_{U}$ transformations and exhibit a singularity at the $T=U$ line.

Here, we will complete this picture by giving more details of the calculation. Lets us denote the function with modular weight 4 under T-duality $F^{(4)}(T)$ and the function with modular weight -2 under $U$-duality and the singularity at $T=U$ by $F^{(-2)}(U)$. Then we must have

$$
F^{(4)}(T)=\frac{j_{T}^{2}}{j(j-1)}, \quad \text { and } \quad F^{(-2)}(U)=\frac{j(U)(j(U)-j(i))}{j_{U}(U)(j(U)-j(T))}
$$

As we can regognize $F^{(4)}(T)$ is the $E_{4}(T)$ function which is part of the basis of the modular forms for the group $S L(2, Z)$. Moreover, $F^{(-2)}(U)$ can be rewritten in turn as

$$
F^{(-2)}=\frac{1}{j(T)-j(U)}\left\{-\frac{j_{U}^{2}(U)}{j(U)(j(U)-j(i))}\right\} \frac{j_{U}^{3}(U)}{j(U)^{2}(j(U)-j(i))}\left(\frac{j_{U}^{6}}{j^{4}(j(U)-j(i))^{3}}\right)^{-1}
$$

The terms appearing in eqn.(4.24) represent

$$
F^{(-2)}(U)=\text { const. }\left\{\frac{1}{j(T)-j(U)}\right\} E_{4}(U) E_{6}(U) \eta^{-24}(U)
$$

where the standard theorems of modular forms predict

$$
E_{4}(U) \propto \frac{-j_{U}^{2}(U)}{j(j(U)-j(i))}, E_{6}(U) \propto \frac{J_{U}^{3}(U)}{j^{2}(U)(j(U)-j(i))}, \eta^{24} \propto \frac{j_{U}^{6}(U)}{j^{4}(U)(j(U)-j(i))}
$$


The function $E_{4}$ has a zero at $T=\rho$, and $E_{6}$ has a zero at $T=1$. In addition, $\eta(T)$ is the well known cusp form the Dedekind function. It has a zero at $T=\infty$. Examination of the integral representation of behaviour of $f_{T T T}$ near the point $T=U$, shows that it has a single pole with residue $\frac{-2 i}{\pi}$. This therefore fixes the numerical coefficient in front of $F^{(-2)}(T) \times F^{(4)}(U)$. Together with $F^{-2}(U), F^{4}(T)$, we get the correct result eqn. (4.22).

The prepotential $f_{U U U}$

The prepotential function for the $f_{U U U}$ can be obtained by the replacement $T \leftrightarrow U$ but we prefer to find it from the equation for $f_{U U U}$. By taking appropriate derivatives on (4.15) we find

$$
\partial_{U}^{3} f=f_{U U U}=-i(T-\bar{T})^{2} \partial_{\bar{T}} D_{U} \partial_{U} \mathcal{I}
$$

or equivalently

$$
\partial_{U}^{3} f=i(T-\bar{T})^{2} \partial_{T} D_{U} \partial_{U} \mathcal{I}
$$

Here $D_{U}=\partial_{U}+\frac{2}{U-\bar{U}}$, the covariant derivative with respect to $\mathrm{U}$ variable. It transforms with modular weight 2 under $S L(2, Z)_{U}$ modular transformations, namely

$$
U \stackrel{S L(2, Z)_{U}}{\rightarrow} \frac{a U+b}{c U+d}, \quad D_{U} \rightarrow(c T+d)^{2} D_{U}
$$

We should notice here, that because of the symmetry exchange $T \rightarrow U$, the result for $f_{U U U}$ may come directly from (4.22), by the replacement $T \rightarrow U$. However, this can be confirmed by the solution of (4.27). By using the explicit form of the expression (4.4) and the values of the lattice momenta (4.13), we evaluate the right hand side of (4.27) as

$$
f_{U U U}=8 \pi^{2} \frac{(T-\bar{T})}{(U-\bar{U})^{2}} \int \frac{d^{2} \tau}{\tau_{2}^{2}} \bar{F}(\bar{\tau}) \partial_{\bar{\tau}}\left(\tau_{2}^{2} \partial_{\tau}\left(\tau_{2}^{2} \sum_{P_{L}, P_{R}} P_{L} P_{R} P_{R} P_{R} e^{\pi i \tau\left|P_{L}\right|^{2}} e^{-\pi i \bar{\tau}\left|P_{R}\right|^{2}}\right)\right)
$$

Further integration by parts, with the boundary term vanishing away from the enhanced symmetry points, gives

$$
f_{U U U}=4 \pi^{2} \frac{(T-\bar{T})}{(U-\bar{U})^{2}} \int \frac{d^{2} \tau}{\tau_{2}^{2}} \bar{F}(\bar{\tau}) \sum_{P_{L}, P_{R}} P_{L} P_{R} P_{R} P_{R} e^{\pi i \tau\left|P_{L}\right|^{2}} e^{-\pi i \bar{\tau}\left|P_{R}\right|^{2}} .
$$

Using now, the modular transformations of the momenta

$$
\left(P_{L}, \bar{P}_{R}\right) \stackrel{S L(2, Z)_{T}}{\rightarrow}\left(\frac{c T+d}{c \bar{T}+d}\right)^{\frac{1}{2}}\left(P_{L}, \bar{P}_{R}\right), \quad\left(P_{L}, \bar{P}_{R}\right) \stackrel{S L(2, Z)_{U}}{\rightarrow}\left(\frac{c U+d}{c \bar{U}+d}\right)^{\frac{1}{2}}\left(P_{L}, \bar{P}_{R}\right),
$$


we can see that $f_{U U U}$ transforms correctly, as it should, that is modular weight 4 under $S L(2, Z)_{U}$ and -2 under $S L(2, Z)_{T}$. In addition, we can observe, in analogy with $f_{T T T}$, that (4.31) possesses a simple pole at $T=U$. The $f_{U U U}$ function has similar modular properties, equivalent under the exchange monodromy transformation symmetry of $\mathrm{f}$, and singularity structure as $f_{T T T}$.

As a result, $f_{U U U}$ takes the form

$$
f_{U U U}=\frac{(j(T)-j(i)) j(T)}{j_{T}(T)(j(T)-j(U))} \Psi(U),
$$

with $\Psi(U)$ a meromorphic modular form of weight 4 in $\mathrm{U}$. The $\mathrm{T}$ dependent part of $f_{U U U}$ is a meromorphic modular form of weight -2 and has a singularity at the point $T=U$.

From the integral representation of $f_{U U U}$, eqn. (4.30) we can see that at the limit $T \rightarrow \infty, f_{U U U} \rightarrow 0$, which means that $\Psi(U)$ is holomorphic anywhere. Finally, we get

$$
f_{U U U}=-\frac{2 i}{\pi} \frac{(j(T)-j(i)) j(T)}{j_{T}(T)(j(T)-j(U))} \frac{j_{U}^{2}(U)}{j(U)-j(i)} \frac{1}{j(U)} .
$$

Lets us check the behaviour of (4.34) away and at the fixed points. Away form the fixed points, e.g when $U \rightarrow \hat{T}=\frac{a T+b}{c T+d}, f_{U U U}$ exhibits a singularity

$$
f_{U U U} \rightarrow-\frac{2 i}{\pi} \frac{1}{U-\hat{T}}(c T+d)^{2}
$$

such that the one loop Kähler metric $G_{U \bar{U}}^{(1)}$ behaves exactly as expected²

$$
G_{U \bar{U}}^{(1)} \rightarrow \frac{1}{\pi} \ln |U-\hat{T}|^{2} G_{U \bar{U}}^{(0)}
$$

Exactly, when $\mathrm{T}$ is one of the fixed points of the modular group $S L(2, Z), f_{U U U}$ vanishes. The presence of the logarithmic singulariry in the one loop corrections to $\mathrm{f}$ gives rise to the generation of the discrete shifts in the theta angles due to monodromies around semiclassical singularities in the quantum moduli space where previously massive states become massless 11, 56, 105.

\section{The one loop prepotential f}

The one-loop contribution to the Kähler metric can be calculated through an equation different than eqn.(4.20). Expanding (4.15) appropriately, we get

$$
2 i \mathcal{I}=\partial_{T} \partial_{U} f(T, U)-\frac{2}{T-\bar{T}} f(T, U)-\frac{2}{U-\bar{U}} f(T, U)+\frac{4}{(T-\bar{T})(U-\bar{U})} f(T, U) .
$$

\footnotetext{
${ }^{22}$ From the symmetry enhancement point of view for the $S L(2, Z)$ modular group of the two torus.
} 
Acting with the appropriate derivatives on the left hand of (4.37) the following identity holds:

$$
2 f=i(T-\bar{T})^{2}(U-\bar{U})^{2} \partial_{\bar{U}} \partial_{\bar{T}} \mathcal{I}
$$

or in the symmetric form

$$
2 f=i(T-\bar{T})^{2}(U-\bar{U})^{2} \partial_{\bar{T}} \partial_{\bar{U}} \mathcal{I}
$$

Explicitly,

$$
2 f=i(T-\bar{T})^{2}(U-\bar{U})^{2} \partial_{\bar{U}} \partial_{\bar{T}} \int_{\mathcal{F}} \frac{d^{2} \tau}{\tau_{2}^{2}} \partial_{\bar{\tau}}\left(\tau_{2} Z\right) \bar{F}(\bar{\tau}) .
$$

Eqn. (4.38) is the master equation for the prepotential. It calculates the one loop prepotential of any, rank four, four dimensional $N=2$ heterotic string compactifications. As we can observe, the one loop correction to the holomorphic prepotential comes by taking derivatives of $\mathcal{I}$ with respect to the conjugate moduli variables from which the holomorphic prepotential does not have any dependence. In this way, we always produce the differential equation for the $\mathrm{f}$ function from the string amplitude. In addition, the solution of this equation calculates the one loop correction to the Kähler metric.

The integral representation of (4.38), after using the explicit form of momenta (4.13), is

$$
f=-4 \pi(T-\bar{T})(U-\bar{U}) \int \frac{d^{2} \tau}{\tau_{2}^{2}} \bar{F}(\bar{\tau}) \partial_{\bar{\tau}}\left[\tau_{2}^{2} \partial_{\bar{\tau}}\left(\tau_{2} \sum_{P_{L}, P_{R}} \bar{P}_{L} \bar{P}_{L} e^{i \pi \tau\left|P_{L}\right|^{2}} e^{-i \pi \bar{\tau}\left|P_{R}\right|^{2}}\right)\right]
$$

where we have used the identity

$$
\partial_{\bar{T}} \partial_{\bar{U}} Z=-\frac{4 i \pi \tau_{2}}{(T-\bar{T})(U-\bar{U})} \partial_{\bar{\tau}}\left(\tau_{2} \sum_{P_{L}, P_{R}} \bar{P}_{L} \bar{P}_{L} Z\right)
$$

and the relations

$$
\partial_{\bar{T}} \bar{P}_{L}^{2}=\frac{\bar{P}_{L} P_{R}}{T-\bar{T}}=\partial_{\bar{T}} \bar{P}_{R}^{2}
$$

We can easily see that the one loop prepotential has the correct modular properties, it transforms with modular weight 4 in $\mathrm{T}$ and -2 in U. Eqn.(4.38) is the differential equation that the one loop prepotential satisfies. The solution of this equation determines the one loop correction to the Kähler metric and the Kähler potential for $N=2$ orbifold compactifications of the heterotic string. 
Compactifications of the heterotic string on $K_{3} \times T_{2}$, appears to have the same moduli dependence on $\mathrm{T}$ and $\mathrm{U}$ moduli, for particular classes of models [57, 38, 69, 66]. Formally, the same routine procedure, namely taking the derivatives with respect to the conjugate $T$ and $U$ moduli on $\mathcal{I}$, can be applied to any heterotic string amplitude between two moduli scalars and antisymmetric tensor, in order to isolate from the general solution (4.38) the term $f(T, U)$. The solution for $f_{\text {TтT }}$ in eqn. (4.22) was derived for $N=2$ compactification of the heterotic strings in [57 via the modular properties of the one loop prepotential coming from the study of its integral representation (4.21). Specific application for the model based on the orbifold limit of $K_{3}$, namely $T^{4} / Z_{2}$, equivalent to the $S U(2)$ instanton embedding $(24,0)$, was given in [34]. At the orbifold limit of $K_{3}$ compactification of the heterotic string the Narain lattice was decomposed into the form $\Gamma^{22,6}=\Gamma^{2,2} \oplus \Gamma^{4,4} \oplus \Gamma^{16,0}$. It was modded by a $Z_{2}$ twist on the $T^{4}$ part together with a $Z_{2}$ shift $\delta$ on the $\Gamma^{(2,2)}$ lattice. For reasons of level matching $\delta^{2}$ was chosen to be $1 / 2$. The unbroken gauge group for this model is $E_{8} \times E_{7} \times U(1)^{2} \times U(1)^{2}$. By an explicit string loop calculation via the one loop gauge couplings in [34, from where the one loop prepotential was extracted with an ansatz, they were able to calculate the third derivative of the prepotential. The latter result was found to agree with the corresponding calculation in [56, 57, which was calculated for the S-T-U subspace of the vector multiplets of the orbifold compactification of the heterotic string. Here, we will check part of this result. In particular, we will confirm the moduli dependence coming from the trilogarithm, for the case of $S U(2)$ instanton embeddings [75, 15] $(12,12)$, $(10,14),(11,13)$ and $(24,0)$ for which the index in the Ramond sector takes the same value.

In reality, $\bar{F}(\bar{\tau})$ is the trace of $F^{\prime}(-1)^{F^{\prime}} q^{L_{o}-\frac{c}{24}} \bar{q}^{\bar{L}_{o}}-\frac{c}{24} / \eta(\bar{\tau})^{2}$ over the Ramond sector boundary conditions of the remaining superconformal blocks. For the S-T-U model with instanton embedding $\left(d_{1}, d_{2}\right)=(0,24)$ their supersymmetric index was calculated in 34 in the form

$$
\text { Index }=\frac{1}{\eta^{2}} \operatorname{Tr}_{R} F^{\prime}(-1)^{F^{\prime}} q^{L_{o}-\frac{c}{24}} \bar{q}^{\bar{L}_{o}-\frac{c}{24}}=-2 i \frac{\bar{E}_{4} \bar{E}_{6}}{\bar{\Delta}}, \quad \frac{\bar{E}_{4} \bar{E}_{6}}{\bar{\Delta}}=\sum_{n \geq-1} c_{1}(n) \bar{q}^{n} .
$$

where $F^{\prime}$ is the right moving fermion number. This is exactly, the value of our index in eqn.(4.7) except for our normalization factor of $1 /(2 \pi)^{2}$ which accounts for the linear

\footnotetext{
${ }^{23}$ Especially, for the S-T-U models the contribution to the index from the different instanton embeddings $d_{1}, d_{2}$ in the two $E_{8}$ factors is independent 86 from the specific instanton embeddings.
} 
representation for the dilaton.

Expanding $\mathcal{I}$ we get that

$$
\mathcal{I}=(-i \pi) \int \frac{d^{2} \tau}{\tau_{2}}\left(p_{R}^{2}-\frac{1}{2 \pi \tau_{2}}\right) \bar{F}(\bar{\tau})
$$

We remind here, a general remark, that the index $\bar{F}$ was determined using, the theory of modular forms, its modular properties and singularity structure alone.

Specific tests of dual pairs were performed, in the spirit of [34], in [86, 90, 91, 92, 93]. The low energy $N=1$ supergravity of type I and heterotic string theories is subject to anomalies coming from hexagon diagrams which prevent it from describing an anomaly free string theory. In this case anomalies are cancelled [79, 78, 36] by the addition of appropriate counterterms which modify the supersymmetry structure. Similarly, in six dimensions the total anomaly is associated to the eight form

$$
I_{8}=\tilde{\theta}_{1} \operatorname{tr} R^{4}+\tilde{\theta}_{2}\left(t r R^{2}\right)+\tilde{\theta}_{3} \operatorname{tr} R^{2} \operatorname{tr} F^{2}+\tilde{\theta}_{4}\left(t r F^{2}\right)^{2}
$$

where $\tilde{\theta}_{1}, \tilde{\theta}_{2}, \tilde{\theta}_{3}, \tilde{\theta}_{4}$ are numbers depending on the spectrum [77, 80] of the theory. Cancellation of the anomaly requires $\tilde{\theta}_{1}=n_{H}-n_{V}+29 T-273=0$, where $n_{V}, n_{H}, n_{T}$ are the numbers of vector multiplets, hypermultiplets and antiselfdual tensor multiplets respectively. Because in six dimensions we have one tensor multiplet [58, 60, 59, 61], which incorporates the dilaton, a Weyl spinor and an antiself-dual antisymmetric tensor, the last constraint becomes $n_{H}-n_{V}=244$. Now Green Schwarz mechanism factorization of anomalies is at work with $I_{8} \propto-\mathcal{G} \tilde{\mathcal{G}}, \mathcal{G}=\operatorname{tr} R^{2}-\sum_{a} v_{a}\left(t r F^{2}\right)$ and सै $^{\mathcal{G}}=\operatorname{tr}(R \wedge R)-\sum_{a} \tilde{v}_{a} \operatorname{tr}(F \wedge F)_{a}$. Cancellation of anomalies requires modification of the antisymmetric field stregth $\mathrm{H}$ as

$$
H=d B+\omega^{L}-\sum_{a} v_{a} \omega_{a}^{Y M}, \omega_{L}=\operatorname{tr}\left(\omega R-\frac{1}{3} \omega^{3}\right), \omega^{Y M}=\operatorname{tr}\left(A F-\frac{1}{3} \omega^{3}\right) .
$$

Here, $\omega_{L}, \omega^{Y M}$ are the Yang-Mills and Lorentz Chern-Simons three forms, A the gauge field, $\mathrm{R}$ the Riemann tensor and $\omega$ the spin connection. However, because $\mathrm{H}$ is globally defined on $K_{3}, \int_{K_{3}} d H=0$. As a result, we get that the following constraint has to be satisfied,

$$
\sum_{a} n_{a}=24, n_{a}=\int_{K_{3}} \operatorname{tr} F^{2}, \int_{K_{3}} \operatorname{tr} R^{2}=24
$$

\footnotetext{
${ }^{24}$ Here, R, F are the gravitational and gauge field strengths. The coefficients $v_{a}, \tilde{v}_{a}$ depend on the particle content and the sum is over the gauge group $\mathrm{G}$ factors $G_{a}$.
} 
Here, the instanton number $n_{a}$ becomes equal to the Euler number of $K_{3}$. Intially, in ten dimensions the unbroken group is $E_{8} \times E_{8} \times U(1)^{4}$, where the $U(1)$ 's are associated with the $T^{2}$ and the graviton and the graviphoton. The spectrum of the theory after compactification on $K_{3} \times T^{2}$ can be calculated [77] using index theory [62, 38]. The gauge group $\mathrm{G}$ can be broken to a subgroup $\mathrm{H}$, by vacuum expectation values of $K_{3}$ gauge fields in $\mathcal{G}$, where $H \times \mathcal{G} \subset G$. The gauge group $\mathrm{G}$ breaks into the subroup $\mathrm{H}$, which is the maximal subgroup commuting with the $\mathcal{G}$ subroup, the commutant of $\mathcal{G}$. We perform the decomposition $\operatorname{adj} G=\sum_{i}\left(R_{i}, M_{i}\right)$, where $R_{i}, M_{i}$ representations of the gauge groups $\mathrm{H}$ and $\mathcal{G}$ respectively. Then the number of left-handed spinor multiplets transforming in the $R_{i}$ representation of $\mathrm{H}$ is given by

$$
N_{R_{i}}=\int_{K_{3}}-\frac{1}{2} \operatorname{tr}_{R_{i}} F^{2}+\frac{1}{48} \operatorname{dim}_{M_{i}} \operatorname{tr} R^{2}=\operatorname{dim}_{M_{i}}-\frac{1}{2} \int_{K_{3}} c_{2}(V) \operatorname{index}\left(M_{i}\right),
$$

where $\mathrm{V}$ is the $\mathcal{G}$ bundle parametrizing the expectation values(vev's) of the vacuum gauge fields on $K_{3}$. By $c_{2}(V)$ we denote the second Chern class of the gauge bundle $\mathrm{V}$ and $\operatorname{dim}_{i}$ the dimension of the representation i. In addition, the dimension of the moduli space of gauge bundles is $4 h_{a}-\operatorname{dim}\left(\mathcal{G}_{a}\right)$, where $h_{a}$ is the Coxeter number of $\mathcal{G}_{a}$ and dim its rank. In a general situation we allow for the gauge group $\mathrm{G}$ to break to the commutant of $\otimes \mathcal{G}$, by embedding the gauge connections of a number of a product of gauge bundles $V_{a}$ with gauge group $\mathcal{G}_{a}$ into $\mathrm{G}$, resulting in the breaking of $\mathrm{G}$ into the commutant of $\otimes_{a} \mathcal{G}_{a}$. In this way, we identify, for manifolds of $S U(2)$ holonomy, the spin connection of $K_{3}$ with the gauge group $\otimes_{a} \mathcal{G}_{a}$, breaking the $\mathrm{G}$ symmetry into $\mathrm{H}$. This is the analog of breaking the gauge group $E_{8}$, in manifolds of $S U(3)$ holonomy, by the standard embedding[85] of the $S U(3)$ gauge connection into the spin connection, to the phenomenologically interesting $E_{6}$ gauge group.

Comments on the modular integral calculation

Let us apply eqn.(4.40) for the calculation of prepotential in the S-T-U model. Remember that the prepotential for this model was calculated from an ansatz solution. The index for this model is independent [86] of the $\mathrm{z}$ particular instanton embedding $\left(n_{1}, n_{2}\right)$ in the two $E_{8}$ factors and is equal to (4.44). We set

$$
\frac{E_{4} E_{6}}{\triangle}(\bar{\tau})=\sum_{n \geq-1} c(n) q^{n}=c(-1) q^{-1}+c(0)+\ldots
$$


The $\mathcal{I}$ integral in eqn.(4.38) has been discussed before in 15 . Using the values of the momenta (4.13) in (4.38) and using Poisson resummation we get

$$
\mathcal{I}=(i \pi) T_{2}^{2} \int \frac{d^{2} \tau}{\tau_{2}^{4}} \sum_{n_{1}, n_{2}, l_{1}, l_{2}} Q_{R} \bar{Q}_{R} e^{-2 \pi i \bar{T} \operatorname{det} A} e^{\frac{-\pi T_{2}}{\tau_{2} U_{2}}\left|n_{1} \tau+n_{2} U \tau-U l_{1}+l_{2}\right|^{2}} \bar{F}(\bar{\tau})
$$

where

$$
Q_{R}=\frac{1}{\sqrt{2 T_{2} U_{2}}}\left(n_{2} \bar{U} \tau+n_{1} \tau-\bar{U} l_{1}+l_{2}\right), \quad \bar{Q}_{R}=\frac{1}{\sqrt{2 T_{2} U_{2}}}\left(n_{2} U \tau+n_{1} \tau-U l_{1}+l_{2}\right)
$$

The integral (4.51) can be calculated using the method of decomposition into modular orbits[16, 33] of $\operatorname{PSL}(2, Z)$. There are three contributions to the modular integral. The zero orbit $A=0$, the degenerate orbit and the non-degenerate orbit. The zero orbit $A=0$ gives no contribution to the $\mathcal{I}$ integral. The next orbit that we will examine is the nondegenerate orbit for which the matrix representative is

$$
A= \pm\left(\begin{array}{cc}
k & j \\
0 & p
\end{array}\right), \quad 0 \leq j<k, \quad p \neq 0
$$

This integral has been calculated in 15 and it is given 25 by

$$
\begin{aligned}
& \mathcal{I}=-\frac{1}{(2 \pi)} \sum_{k>0} \sum_{p>0} \delta_{n, k l}\left(\frac{2 k l}{p}+\frac{l}{\pi T_{2} p^{2}}+\frac{k}{\pi U_{2} p^{2}}+\frac{1}{2 \pi^{2} T_{2} U_{2} p^{3}}\right) x^{p}+h . c, \\
& l \in Z
\end{aligned}
$$

where $x \stackrel{\text { def }}{=} e^{2 \pi i(k T+l U)}$ and $\bar{x} \stackrel{\text { def }}{=} e^{-2 \pi i(k \bar{T}+l \bar{U})}$. Substituting eqn.(4.54) in the master equation for the prepotential (4.38) we get that the contribution of the orbit $\mathcal{I}_{1}$ in $\mathrm{f}$ is

$$
\left.f\right|_{\text {non-degenerate }}=(2 i)\left(\frac{2}{(2 \pi)^{3}} \sum_{(k, l)>0} c(k l) \mathcal{L} i_{3}\left[e^{2 \pi i(k T+l U)}\right]\right) .
$$

This is exactly the moduli dependence on the trilogarithm found indirectly in [34. The dependence of the solution in $i$, out of the parenthesis in (4.55) is neccessary since it is used to cancel the overall dependence on $\mathrm{i}$ in the one loop Kähler metric (4.12). Note that in the previous equation we have not considered the complex conjugate solutions which arise by taking the partial derivatives with respect to the $\bar{T}$ and $\bar{U}$ variables in the complex

${ }^{25}$ After proper incorporation of the normalization factors of our Ramongd index. 
conjugate part of the solution of eqn.(4.54). There are two ways to see this. One is the mathematical point of view while the other clearly come from physical requirements. The physical point is that the prepotential has to be a holomorphic function of the vector moduli variables. On the other hand, the integral $\mathcal{I}$, which comes as a solution of the one loop Kähler metric in eqn.(4.12), includes the complex conjugate part of the action of the two covariant derivatives on the prepotential $\mathrm{f}$. However, the solution for the prepotential as was defined here in eqn.(4.38) comes from the general solution of the Kähler metric which does not include the conjugate part of its solution. Results of the integration coming from the degenerate orbit and related matters will appeal elsewhere[49].

\section{Application to rank three $N=2$ heterotic string compactifi- cations}

We have said that one important aspect of the expected duality is that the vector moduli space of the heterotic string must coincide at the non-perturbative level with the tree level exact vector moduli space of the type IIA theory. For the type IIA superstring compactified on a Calabi-Yau space X the internal $(2,2)$ moduli space has $N=2$ worldsheet supersymmetry for the left and the right movers and is described, at the large complex structure limit of $\mathrm{X}$, by the Kähler moduli, namely $B+i J \in H^{2}(X, C)$, where $B+i J=$ $\sum_{i=1}^{h(1,1)}(B+i J)_{a} e_{a}$ with $B_{a}, J_{a}$ real numbers and $t_{a}=(B+i J)_{a}$ representing the special coordinates and $e_{a}$ a basis of $H^{2}(X, C)$.

In this section we will derive the general form of the equation determining the prepotential form the rank three $N=2$ heterotic compactifications. In particular we will examine a type II model admitting a heterotic perturbative dual realization. The heterotic model contains three moduli the dilaton $\mathrm{S}$, the graviphoton, and one moduli the $\mathrm{T}$ moduli. It coincides with the corresponding Calabi-Yau dual model at its weak coupling limit.

In order for the heterotic prepotential to match its Calabi-Yau dual at its weak coupling

\footnotetext{
${ }^{26}$ Let us consider the target space of a complex manifold $\mathcal{M}$ with dimension n. Choose coordinates on $\mathcal{M}, \phi_{m}$ and $\bar{\phi}_{m}$. Then $\mathcal{M}$ admits a a Kähler structure if we can define a $(1,1)$ form $\mathrm{J}$ with the property $J=i G_{l \bar{m}} d \phi_{m} \wedge d \bar{\phi}_{l}$ where for a Kähler manifold the metric is $G_{l \bar{m}}=\partial_{\phi^{m}} \partial_{\bar{\phi}_{l}} K$, and the Kähler potential is $\mathrm{K}$.
} 
limit a number of conditions are necessary, which we will briefly review them here. The existence of a type II dual to the weak coupling phase of the heterotic string is exactly the existence of the conditions 74

$$
D_{s s s}=0, \quad D_{s s i}=0 \text { for every } i,
$$

where D the Calabi-Yau divisors appearing in (5.4). An additional condition originates from the higher derivative gravitational couplings of the heterotic vector multiplets and the Weyl multiplet of conformal $N=2$ supergravity[83]. The relevant couplings originate from terms in the form $g_{n}^{-2} R^{2} G^{2 n-2}$, where $\mathrm{R}$ is the Riemann tensor, $\mathrm{G}$ the field strength of the graviphoton. The $g_{n}$ couplings obey $g_{n}^{-2}=\operatorname{Re} \tilde{F}_{n}\left(S, M^{i}\right)+\ldots$. The same of couplings appear in type II superstring 84. In the heterotic side they take the form

$$
\tilde{F}_{n}=\tilde{F}^{(0)}\left(S, M_{i}\right)+\tilde{F}^{1}\left(M^{i}\right)+\tilde{F}^{N P}\left(e^{-8 \pi^{2} S}, M^{i}\right), \quad \tilde{F}_{1}=24 S, \quad \tilde{F}_{n \geq 1}^{o}=\text { const },
$$

where $\mathrm{S}$ is the heterotic dilaton and $M^{i}$ the rest of the vector multiplets moduli. Such terms appear as well in the effective action of type II vacua and they have to match with heterotic one's due to duality. In the large radius limit the higher derivative couplings satisfy(the lowest order coupling) $\tilde{F}_{1} \rightarrow-\frac{4 \pi i}{12} \sum_{a}\left(D_{a} \cdot c_{2}\right) t_{a}$, where $c_{2}$ is the second Chern class of the three fold X. Because at the tree level, $g_{1}^{2}=R e \tilde{F}_{1}$ we can infer the result that

$$
D_{a} \cdot c_{2}(X)=24
$$

The last condition represents 74 the mathematical fact that the Calabi-Yau threefold X admits a fibration $\Phi$ such as there is a map $X \rightarrow W$, with the base being $P^{1}$ and generic fiber the $K_{3}$ surface. Furthermore, the area of the base of the fibration gives the heterotic four dimensional dilaton.

In the content of the moduli of the Calabi-Yau space of $\mathrm{X}$, the holomorphic prepotential at the large radius limit takes the form

$$
F=-\frac{i}{6} \sum_{\alpha, \beta, \gamma}\left(D_{\alpha} \cdot D_{\alpha} \cdot D_{\gamma}\right) t_{\alpha} t_{\beta} t_{\gamma}-\frac{\chi \zeta(3)}{2(2 \pi)^{3}} \sum_{\left(d_{i}\right)_{i=1, \ldots, n}} n_{d_{1}, \ldots, d_{n}} \mathcal{L} i_{3}\left(\Pi_{i=1}^{n} q_{i}^{d_{i}}\right),
$$

where the trilogarithmic function is $\mathcal{L} i_{3}(x) \stackrel{\text { def }}{=} \sum_{j \geq 1} \frac{x^{j}}{j^{3}}$. The first term in eqn.(5.4) is a product of the the Calabi-Yau divisors $\mathrm{D}$, associated to the basis $e_{a}$, and the second 
term 8] represents world-sheet instanton contributions. The $n_{d_{1}, \ldots, d_{n}}$ are the world sheet instanton numbers, the numbers of genus zero rational curves, and $d_{i}$ the degrees of the curves. Performing duality tests, at the weak coupling heterotic limit, between a heterotic model and its possible type IIA dual is then equivalent to comparing the weak coupling limit of the prepotential 38 of the vector multiplets for the heterotic string with the large radius limit of (5.4). After identifying the heterotic dilaton with one of the vector moduli of the type IIA model in the form $t_{s}=(B+i J)_{s}=4 \pi i S$, the type IIA prepotential takes the general form 43.

$$
\mathcal{F}_{I I A}=-\frac{1}{6} C_{A B C} t^{A} t^{B} t^{C}-\frac{\chi \zeta(3)}{2(2 \pi)^{3}} \sum_{d_{1} \ldots, d_{n}} n_{d_{1}, \ldots, d_{n}} \mathcal{L}_{i_{3}}\left[e^{2 \pi i\left[\sum_{k} d_{k} t^{k}\right]}\right],
$$

where we are working inside the Kähler cone 8, 71 $\sigma \stackrel{\text { def }}{=}\left\{\sum_{\rho} t^{\rho} J_{\rho} \mid t^{\rho}>0\right\}$, where $J^{\rho}$ generate the cohomology group $H^{2}(X, R)$ of the Calabi-Yau three fold X.

In 69 it was noticed that the nature of type II-heterotic sting duality test has to come from the $K_{3}$ fiber structure over $P^{1}$ of the type IIA side. The form of the $K_{3}$ fibration can be found 69, 66] by taking for example the CY in $P^{4}\left(1,1,2 k_{2}, 2 k_{3}, 2 k_{4}\right)$ and then set $x_{o}=\lambda x_{1}$. After rescaling $x_{1} \rightarrow x_{1}^{1 / 2}$ we arrive at the equation for the hypersurface

$$
F(\lambda) Z_{1}^{d}+Z_{2}^{d / k_{2}}+\cdots=0
$$

The degree $d=1+k_{2}+k_{3}+k_{4}$. For generic values of $\lambda$ eq.(5.6) is a $K_{3}$ surface in weighted $P^{3}$. So $P^{4}\left(1,1,2 k_{2}, 2 k_{3}, 2 k_{4}\right)$ is a $K_{3}$ fibration fibered over the $P^{1}$ base which is parametrized by $\lambda$. At the large radius limit of $\mathrm{X}$ the heterotic dilaton $\mathrm{S}$ is identified as one of the vector multiplet variables as $t_{s}=4 \pi i S$. Confirmation of duality between dual pairs is then equivalent to the identification 37

$$
\mathcal{F}_{I I A}=\mathcal{F}_{I I A}\left(t^{s}, t^{i}\right)+\mathcal{F}_{I I A}\left(t^{i}\right)=\mathcal{F}_{\text {het }}^{o}\left(S, \phi^{I}\right)+\mathcal{F}_{\text {het }}^{(1)}\left(\phi^{I}\right) .
$$

Here, we have expand the prepotential of the type IIA in its large radius limit, namely large $t_{s}$. In the heterotic side, we have the tree level classical contribution as a function of the dilaton $\mathrm{S}$ and the vector multiplet moduli $\Phi^{I}$, in addition to the one loop correction as a function of only the $\Phi^{I}$.

Dual pairs for which the prepotential in the type IIA theory is known can be mapped to the type IIB using mirror symmetry 76 . Let us review some aspects of the low energy 
theory of the type IIB superstrings. In Calabi-Yau manifolds, special geometry is associated with the description of their moduli spaces. In type IIB, the $H^{2,1}$ cohomology describes the deformation of the complex structure of the Calabi-Yau space $\mathcal{M}$. Now the Kähler metric for the $(2,1)$ moduli is defined $\left[27\right.$ from the Weyl Peterson metric 8, 43, 44, 64] $\sigma_{i j}$, namely

$$
G_{i j}=\sigma_{i j} /\left(i\left(\int_{\mathcal{M}} \Omega \wedge \bar{\Omega}\right)\right)
$$

where

$$
\varphi_{i}=(1 / 2) \varphi_{i k \lambda \bar{\rho}} d x^{k} d x^{k} d x^{\lambda} d x^{\bar{\rho}}, \quad \sigma_{i j}=\int_{\mathcal{M}} \varphi_{i} \wedge \bar{\varphi}_{\bar{j}}
$$

and $\varphi_{i k \lambda \bar{\rho}}=\left(\partial g_{\bar{\rho} \bar{\xi}} / \partial t^{i}\right) \Omega_{k \lambda}^{\bar{x} i}$. Here, $t_{i}=1, \ldots, b_{2,1}$ and $G_{i j}=-\partial_{i} \partial_{j}\left(i \int_{\mathcal{M}} \Omega \wedge \bar{\Omega}\right)$. The three form tensor $\Omega$ is given in terms of the homology basis $\alpha, \beta$ as $\Omega=X^{I} \alpha_{I}+i F_{I} \beta^{I}$. The complex structure is described by the periods of the holomorphic three form $\Omega$ over the canonical homology basis. Here, the periods are given by $X^{I}=\int_{A^{I}} \Omega, i F_{I}=\int_{B^{I}} \Omega$ the integral of the holomorphic three form over the homology basis. The Kähler potential comes from the moduli metric

$$
G_{i j}=-i \partial_{i} \partial_{j}\left\{i \int \Omega \wedge \bar{\Omega}\right\}, K=-\log \left(X^{I} \bar{F}_{I}+\bar{X}^{I} F_{I}\right)
$$

Now the Riemmann tensor is defined as

$$
R_{i \bar{j} k \bar{l}}=G_{i \bar{j}} G_{k \bar{l}}+G_{i \bar{l}} G_{k \bar{j}}-\overline{\mathcal{C}}_{i k n} \overline{\mathcal{C}}_{\bar{j} \bar{l} \bar{n}} G^{n \bar{n}} e^{2 K}
$$

where the expression of the Yukawa couplings in a general coordinate system are given by $\mathcal{C}=\int \Omega \wedge \partial_{i} \partial_{j} \partial_{k} \Omega, \partial_{i}=\partial / \partial z^{i}$. The holomorphic function $\mathrm{F}$ does not receive quantum corrections from world-sheet instantons and as a consequence neither the the Kähler potential derived from it. Calabi-Yau threefolds can be constructed[118] among other ways as a hypersurface or as a complete intersection of hypersurfaces in a weighted projective space $P^{N}(\vec{w})$. Remember, that the complex projective space $C P^{N}$ is the space defined by the homogeneous complex coordinates $Z_{1}, \ldots, Z_{N+1}$ which obey $\left(Z_{1}, \ldots, Z_{N+1}\right) \stackrel{\lambda \neq 0}{=}$ $\left(\lambda^{w_{1}} Z_{1}, \ldots, \lambda^{w_{n+1}} Z_{N+1}\right)$ for complex $\lambda$. The threefold is obtained from the $C P^{4}$, while the $K_{3}$ can be obtained from the $\sum_{k_{i}} \alpha_{k_{1} k_{2} k_{3} k_{4}} x_{k_{1}} x_{k_{2}} x_{k_{3}} x_{k_{4}}=0$, in projective $P^{3}$ and $P^{2}$ respectively(rp). They describe complex manifolds parametrized by 135 and 35 complex coefficients $a_{k_{i}} \mathrm{rp}$, which after removing an overall redundancy they give 101, 19 elements of

\footnotetext{
${ }^{27}$ In the rest of the section the notation for the special coordinates is as follows, $Z^{i}=-i X^{i} / X^{o}$.
} 
$H^{(1,1)}$ rp. The weighted projective space $P^{N}(\vec{w})$ is defined by the conditions on the homogeneous coordinates $\left(Z_{1}, \ldots, Z_{N+1}\right) \stackrel{\lambda \neq 0}{=}\left(\lambda^{d_{1}}, \ldots, \lambda^{d_{N+1}}\right)$ and $P^{N}\left(\lambda^{d_{1}} Z_{1}, \ldots, \lambda^{d_{N+1}} Z_{N+1}\right) \stackrel{\text { def }}{=}$ $\left\{C^{N+1} /\left(Z_{1}=0, \ldots, Z_{N+1}=0\right)\right\}$. The last condition, excludes the origin of the complex space. The $d_{i}$ are the weights and the sum of the weights is the degree of the variety.

Let us consider the Calabi-Yau three fold defined as the zero locus of the hypersurface $P_{1,1,2,2,2}^{4}$ of degree eight. This model appears in the list of [38] as the A model and it is defined as $X_{8}(1,1,2,2,2)_{2}^{-86}$, where the subscripts and superscripts denote the Betti numbers $b_{1,1}=2$ and $b_{1,2}=86$. This model gives rise to 2 vector multiplets and $86+$ 1 hypermultiplets including the dilaton and its moduli space can be studied using mirror symmetry [76, 72].

The mirror manifold $X_{8}^{*}$ for this model is defined by the Calabi-Yau three fold in the form $\{\mathcal{P}=0\} / Z_{4}^{3}$, where the zero locus is

$$
\mathcal{P}=z_{1}^{8}+z_{2}^{8}+z_{3}^{4}+z_{4}^{4}+z_{5}^{4}-8 \psi z_{1} z_{2} z_{3} z_{4} z_{5}-2 \phi z_{1}^{4} z_{2}^{4}
$$

It depends on the deformation parameters $\phi$ and $\psi$. The $Z_{4}^{3}$ symmetry acts on the coordinates as $\left(z_{2}, z_{2+m}\right) \rightarrow\left(-i z_{2}, i z_{2+m}\right)$ for $m=1,2,3$, respectively. A good description of the moduli space is obtained by enlarging the group $\{\mathcal{P}=0\} / Z_{4}^{3}$ to the group $\hat{G}$ acting as

$$
\left(z_{1}, z_{2}, z_{3}, z_{4}, z_{5} ; \psi, \phi\right) \rightarrow\left(\omega^{\tilde{a}_{1}} z_{1}, \omega^{\tilde{a}_{2}} z_{2}, \omega^{2 \tilde{a}_{3}} z_{3}, \omega^{2 \tilde{a}_{4}} z_{4}, \omega^{2 \tilde{a}_{5}} z_{5} ; \omega^{-\tilde{a}} \psi, \omega^{-4 \tilde{a}} \phi\right)
$$

where $\tilde{a}=e^{2 \pi i / 8}, \tilde{a}_{i}$ are integers such as $\tilde{a}=\tilde{a}_{1}+\tilde{a}_{2}+2 \tilde{a}_{3}+\tilde{a}_{4}+\tilde{a}_{5}$. Modding the weighted projective spaces by the group $\hat{G}$ requires modding out by the action $(\phi, \psi) \rightarrow(-\phi, \tilde{\alpha} \psi)$. The prepotential of the type IIB model defined on the mirror manifold $X_{8}^{*}$ was calculated in [98 form the study of the Yukawa couplings in [8, 70, 71] as

$$
\mathcal{F}^{I I}=-2 t_{1}^{2} t_{2}-\frac{4}{3} t_{1}^{3}+\ldots+f^{N P} .
$$

From the form of the prepotential we can infer that the type II model has a heterotic dual which corresponds to the particular identification of $t_{2}$ with the heterotic dilaton and $t_{1}$ with the heterotic T moduli. As a result

$$
f^{\text {heterotic }}=-2 S T^{2}+f(T)+f^{\text {non-pertur }}
$$

where $f(T)$ the one loop correction and $f^{\text {non-pertur }}$ the non-perturbative contributions. The heterotic model is an S-T model, a two moduli example or rank three model, if someone 
takes into account the graviphoton. The exact correspondence of $P_{1,1,2,2,2}^{4}$ with the three rank heterotic model is their connection via their classical T-duality group. The two models at the weak coupling limit of the $t_{2}$ moduli have the same classical duality group, $\Gamma_{o}(2)_{+}$. Study of the discriminant of $P_{1,1,2,2,2}^{4}$ gives that the conifold singularity should correspond to the perturbative $S U(2)$ enhanced symmetry point. This fixes the momenta for the $\Gamma^{(2,1)}$ compactification lattice of the heterotic model as

$$
p_{L}=\frac{i \sqrt{2}}{T-\bar{T}}\left(n_{1}+n_{2} \bar{T}^{2}+2 m \bar{T}\right), \quad p_{R}=\frac{i \sqrt{2}}{T-\bar{T}}\left(n_{1}+n_{2} T \bar{T}+m(T+\bar{T})\right) .
$$

with the enhanced symetry point at level 2. Let me discuss first the master equation for the general rank three model, as well the dual heterotic of $P_{1,1,2,2,2}^{4}$. The solution for the one loop correction to the Kähler metric reads 98

$$
K_{T \bar{T}}=K_{T \bar{T}}^{(o)}\left\{1+\frac{2 i}{S-\bar{S}} \mathcal{I}+\ldots\right\} .
$$

Using now the general form of solution for the Kähler metric

$$
\mathcal{I}=\frac{i}{8}\left(\partial_{T}-\frac{2}{T-\bar{T}}\right)\left(\partial_{T}-\frac{4}{T-\bar{T}}\right) f(T)+h . c,
$$

we can infer the master equation for the perturbative one loop correction to the prepotential as

$$
2 i f(T)=(T-\bar{T})^{3} \partial_{\bar{T}} \mathcal{I}
$$

Here $K_{T \bar{T}}^{(o)}$ is the tree level metric $-2 /(T-\bar{T})^{2}$ and $\bar{C}_{l}(\bar{\tau})$ is the index of the Ramond sector in the remaining superconformal blocks. Note that eqn.(5.19) was derived from the general solution for the one loop Kähler metric without any reference to values of momenta for the $\Gamma^{(2,1)}$. This means that this equation determines the prepotential for any rank three $N=2$ compactification of the heterotic string. For example (5.19) determines the heterotic duals of the models B, C in [69, with associated modular groups $\Gamma_{o}(3)_{+}, \Gamma_{o}(4)_{+}$and enhanced symmetry points at, the fixed points of their associated modular groups, Kac-Moody levels 3 and 4 respectively 120 .

The one loop Kähler metric[10, 98] for the heterotic model dual to the type $P_{1,1,2,2,2}^{4}$ model reads

$$
\mathcal{I}=\sum_{i=1}^{6} \int \frac{d^{2} \tau}{\tau_{2}^{3 / 2}} \bar{C}_{l}(\bar{\tau}) \partial_{\bar{\tau}}\left(\tau_{2}^{1 / 2} \sum_{p_{L}, p_{R} \in \Gamma_{l}} e^{\pi i \tau\left|p_{L}\right|^{2}} e^{-\pi i \bar{\tau} p_{R}^{2}}\right)
$$


Direct substitution of the values of the $\Gamma^{(2,1)}$ compactification lattice momenta in (5.20) may give us the prepotential $\mathrm{f}$ in its integral representation. Here the sum is over 98 the different lattice sectors $\Gamma_{l}$,

$$
\begin{array}{ccc}
\Gamma_{1, \epsilon} & \Gamma_{2, \epsilon} & \epsilon=1,2 \\
\Gamma_{1, \epsilon} & n_{1} \in Z+\frac{1}{2} & n_{2} \text { odd } \\
\Gamma_{2, \epsilon} & n_{1} \in Z & n_{2} \text { even } \\
\Gamma_{3, \epsilon} & n_{1} \in Z+\frac{n_{2}+1}{2} & n_{2} \in Z,
\end{array}
$$

where $m \in Z+\epsilon$, that are needed due to world-sheet modular invariance.

\section{Conclusions}

We have calculated the general equation which calculates directly the one loop perturbative prepotential of $N=2$ heterotic string compactifications for any rank three or rank four parameter models. These heterotic string compactifications may or may not have a type II dual compactified on a Calabi-Yau. In general, heterotic vacua with instanton embeddings numbers $(12-n, 12+n)$ on the $E_{8} \times E_{8}$ gauge bundle are associated [41, 75] to the elliptic fibrations over the Hirzebrush surfaces $F_{n}$. Especially, for the families of Calabi-Yau threefolds with Hodge numbers $(3,243)$, associated with $K_{3}$ fibrations and elliptic fibrations, when $\mathrm{n}$ is even the rank three Calabi-Yau is an elliptic fibration over the Hirzebrush surface $F_{2}$ or $F_{0}$, while for n odd the rank three models are given in terms of the Hirzebrush surface $F_{1}$. At the heterotic perturbative level all the models, which are coming from complete Higgising of the charged hypermultiplets, with the same Hodge numbers, come from the instanton embeddings $(12,12),(11,13),(10,14)$. However, at the heterotic perturbative level all the models are the same as we have already said. This is clearly seen from the nature of the Ramond index (4.7) which is independent from the particular instanton embedding. In particular, we tested the moduli dependence of the prepotential, coming from the non-degenerate orbit, for the previous $S U(2)$ instanton embeddings, and the $(24,0)$ one, against the moduli dependence of the prepotential extracted from the one loop corrections to the gauge couplings in [34]. In addition, we calculated the differential equation of the third derivative of the prepotential for the rank four S-T-U model with respect of the complex structure $\mathrm{U}$ variable. 
The master equation's (4.38), (5.19) open the way for direct testing of the web of dualities, e.g the duality between type I and $K_{3} \times T^{2}$ at their weakly coupled region which was tested via the third derivatives of the one loop prepotential in 121]. However, there are other dualities which can be tested at the quantum level. For example, if we continue [9], further compactification on $S^{1}$ of F-theory defined on the elliptic Calabi-Yau, we get duality between M-theory on the associated Calabi-Yau three fold and heterotic strings on $S^{1}$. Further compactification, we get duality between type IIA on Calabi-Yau three folds and heterotic on $K_{3} \times T^{2}$. Furthermore, the direct way of calculating the holomorphic prepotential in (4.38), (5.19) can calculate the $N=2$ central charge and $N=2$ BPS spectrum as well the black hole entropy 122, 123].

\section{Acknowledgments}

We are grateful to I. Antoniadis, C. Kounnas, K. S. Narain, S. Stieberger, D. Zagier and R. Lewes for useful conversations and D. Bailin for reading the manuscript.

\section{Appendix A}

\section{A1 Useful relations with modular forms}

The functions $E_{4}, E_{6}$ form the basis of modular forms for the group $S L(2, Z)$ and are defined in term of Eisenstein series of weight four and six. Namely,

$$
E_{2 k}(T)=\sum_{n_{1}, n_{2} \in Z}^{\prime}\left(i n_{1} T+n_{2}\right)^{-2 k}, \quad k \in Z .
$$

Here the prime means that $n_{1} \neq 0$ if $n_{2}=0$. Let us provide some useful relations between the basis for modular forms for $P S L(2, Z)$ and $\triangle$ and the $\mathrm{j}$ invariant. With the use of these relations various results appeared in the literature, like those in [56], [57] can be easily translated to each other. It can be proved that the following relations hold

$$
\begin{gathered}
E_{4}(T)=-\frac{\left(j^{\prime}\right)^{2}}{4 \pi^{2} j(j-j(i))}=1+240 \sum_{n=1}^{\infty} \sigma_{3}(n) e^{2 \pi i T}, \\
E_{6}(T)=\frac{\left(j^{\prime}\right)^{3}}{(2 \pi i)^{3} j^{2}(j-j(i))}=1-504 \sigma_{5}(n) q^{n}, \\
\triangle(T)=-\frac{1728\left(j^{\prime}\right)^{6}}{\left(48 \pi^{2}\right)^{3} j^{4}(j-j(i))^{3}}=\eta^{24}(T)=\frac{1}{(2 \pi i)^{6}} \frac{\left(j^{\prime}\right)^{6}}{j^{4}(j-j(i))^{3}},
\end{gathered}
$$


where $\eta(T)$ is the Dekekind function and the value of $\sigma$ represents the sum over divisors

$$
\sigma_{h-1}(n) \stackrel{\text { def }}{=} \sum_{d / n} d^{h-1}
$$

We have used the notation

$$
j^{\prime}(T)=j_{T}(T)
$$

Note that in general $E_{4}, E_{6}$ and $\triangle$ are defined in terms of the Klein's absolute invariant J as

$$
\begin{gathered}
j(T) \stackrel{\text { def }}{=} 1728 J(T) \\
J(T)=\frac{E_{4}^{3}(T)}{1728 \triangle(T)}=1+\frac{E_{6}^{2}(T)}{1728 \triangle(T)}, ; \quad T \in H
\end{gathered}
$$

and

$$
j(T)=e^{-2 \pi i T}+744+196884 e^{2 \pi i T}+\ldots
$$

Remember that the following relations are valid

$$
j(T)=\frac{E_{4}^{3}(T)}{\triangle(T)},
$$

and

$$
j(T)=\frac{E_{6}^{2}(T)}{\triangle(T)}
$$

Here, $\mathrm{j}$ is the modular invariant function for the inhomogeneous modular group $P S L(2, Z)$. 


\section{References}

[1] N. Seiberg and E. Witten; Nucl. Phys.B426 (1994) 19;

Erratum B430 (1994) 485; B431 (1994) 484.

[2] A. Klemm, W. Lerche, S. Theisen and S. Yankielowicz;

Phys. Lett. B344 (1995) 169, P. Argyres and A. Faraggi,

Phys. Rev. Lett. 73 (1995) 3931.

[3] L. Dixon, in Proc. of the 1987 ICTP Summer Workshop In High Energy Physics and Cosmology, Trieste, Italy, ed. G. Furlan, J. C. Pati, D. W. Sciama, E. Sezgin and Q. Shafi.

[4] D. Gepner, Phys. Lett. 199B (1987) 380; Nucl. Phys. B296 (1988) 757.

[5] Y. Kazama and H. Suzuki, Phys. Lett. B216 (1989) 112;

Nucl. Phys. B321 (1989) 232.

[6] S. Hamidi and C. Vafa, Nucl. Phys. B279 (1987) 465; L. Dixon, D. Friedan, E. Martinec and S. Shenker, Nucl. Phys. B282 (1987) 13.

[7] R. Dijkgraaf, E. Verlinde and H. Verlinde, Com. Math. Phys. 115 (1988) 669; On Moduli Spaces of Conformal Field Theories with $c \geq 1$, Proceedings Copenhagen Conference,Perspectives in String Theory, ed. by P. Di Vecchia and J. L. Petersen, World Scientific, Singapore, 1988.

[8] P. Candelas, X. de la Ossa, P. Green and L. Parkes, Phys. Lett. B258 (1991) 118; Nucl. Phys. B359 (1991) 21.

[9] C. Vafa, Lectures on Strings and Dualities, hep-th/9702201.

[10] I. Antoniadis, E. Gava and K. S. Narain and T. R. Taylor, Nucl. Phys. B407 (1993) 706.

[11] V. Kaplunovsky and J. Louis, Nucl. Phys. B444 (1995) 501. 
[12] P. Mayr and S. Stieberger, Nucl. Phys. B407 (1993) 725;

D. Bailin, A. Love, W. A. Sabra and S. Thomas, Mod. Phys. Letters. A9 (1994) 67; A10 (1995) 337.

[13] J. Erler and A. Klemm, Commun. Math. Phys. 153 (1993) 579.

[14] H. Ooguri and C. Vafa, Nucl. Phys. B361 (1991) 469.

[15] K. Foerger and S. Stieberger, " String Amplitudes and $N=2 d=4$ Prepotential in Heterotic $K 3 \times T^{2}$ Compactifications", hep-th/9709004.

[16] L. Dixon, V. Kaplunovsky and J.Louis, Nucl. Phys. 355 (1991) 649.

[17] L. Dixon, J. Harvey, C. Vafa and E. Witten, Nucl. Phys. B261 (1985) 678; Nucl. Phys. B274 (1986) 285.

[18] L. E. Ibáñez, J. Mas, H. P. Nilles and F. Quevedo, Nucl. Phys. B301 (1988) 157.

[19] V. Kaplunovsky and J. Louis, Nucl. Phys. B422 (1994) 54.

[20] P. Candelas, X. de la Ossa, A. Font, S. Katz and D. R. Morrison, Nucl. Phys. B416 (1994) 481.

[21] M. A. Shifman and A. I. Vainstein, Phys. Lett. B359 (1991) 571

[22] M. A. Shifman and A. I. Vainstein, Nucl. Phys. B277 (1986) 456

[23] H. P. Nilles and S. Stieberger, Phys.Lett. B367 (1996) 126.

[24] T. M. Apostol, Modular functions and Dirichlet series, Springer 1976.

[25] J. Louis, J. Sonnenschein, S. Theisen, S. Yankielowicz, Nucl. Phys. B480 (1996) 185.

[26] N. Koblitz, 'Introduction to Elliptic curves and modular forms', Springer Verlag, (1983).

[27] L. I. Ibanez, D. Lust, W. Lerche and S. Theisen. Nucl. Phys. B352 (1991) 435. 
[28] I. Antoniadis, C. Bachas and C. Kounnas, Phys. Lett. B200 (1988)297.

[29] D. Bailin, A. Love, W. Sabra and S. Thomas; Phys. Lett. B320 (1994) 21.

[30] D. Bailin, A. Love, W. Sabra and S. Thomas, Mod.Phys. Lett. A9 (1994) 1229.

[31] G. L. Cardoso, D. Lust and T. Mohaupt, Nucl. Phys. B432 (1994) 68.

[32] S. Ferrara, L. Girardello and H. P. Nilles, Phys. Lett. B125 (1983) 457.

[33] E. Kiritsis and C. Kounnas, Nucl. Phys. B442 (1995) 472.

[34] J. Harvey and G. Moore, Nucl. Phys. B463 (1996) 315.

[35] S. Ferrara, C. Kounnas, D. Lust and F. Zwigner, Nucl. Phys. B365 (1991) 431.

[36] P. Candelas, G. Horowitz, A. Strominger and E. Witten, Nucl. Phys. B258 (1985) 46.

[37] J. Louis and K. Foerger, Holomorphic couplings in string theory, Nucl. Phys. Proc. Suppl. 55B (1997) 33.

[38] S. Kahru and C. Vafa, Nucl. Phys B450 (1995) 69.

[39] S. Ferrara, J. A. Harvey, A. Strominger, C.Vafa, Phys. Lett. B361 (1995) 59.

[40] A. Strominger, Nucl. Phys. B451 (1995) 96.

[41] C. Vafa, Nucl. Phys. B469 (1996) 403.

[42] B. Greene, D. Morrison and A. Strominger, Nucl. Phys. B451 (1995) 109.

[43] P. Candelas, P. Green, and T. Hubsch, Nucl. Phys. B330 (1990) 49.

[44] T. M. Chiang, B. R. Green, M. Gross, and Y. Kanter, Nucl. Phys. Proc. Suppl. B46 (1996) 248.

[45] C. Callan, J. Harvey and A. Strominger, Nucl. Phys. B367 (1991) 60.

[46] G. Horowitz and A. Strominger, Nucl. Phys. B360 (1991) 197. 
[47] A. Strominger, Phys. Lett. B383 (1996) 44.

[48] P. Aspinwall and D. Morrison, U-duality and Integral structures, Phys. Lett. B355 (1995) 141.

[49] C. Kokorelis, In preparation.

[50] P. Aspinwall, Enhanced gauge symmetries and $K_{3}$ surfaces, Phys. Lett. B357 (1995) 329.

[51] C. Hull and P. Townsend, "Unity of Superstring dualities", Nucl. Phys. B438 (1995) 109.

[52] A. Sen, "String-String Duality Conjecture in Six Dimensions and Charged Solitonic Strings" , Nucl. Phys. B450 (1995) 103.

[53] E. Witten, Nucl. Phys. B443 (1995) 85.

[54] J. Harvey and A. Strominger, Nucl. Phys. B449 (1995) 535.

[55] for a clear exposition of these issues see: A. Klemm, W. Lerche and S. Theisen: Non perturbative effective actions of $N=2$ Supersymmetric Gauge theories, hep-th/9505150.

[56] B. de Wit, V. Kaplunovsky, J. Louis and D. Lust, Nucl. Phys. B451 (1995) 53.

[57] I. Antoniadis, S. Ferrara, E. Gava, S. Narain, T. R. Taylor, Nucl. Phys. B447 (1995) 35.

[58] M. Sohnius, K. Stelle and P. West, Phys. Lett. B92 (1980) 123.

[59] A. Hindawi, B. Ovrut and D. Waldram, Phys. Lett. B392 (1997) 85.

[60] P. Claus, B. de Wit, M. Faux, B. Kleijn, R. Siebelink and P. Termonia, Phys. Lett. B373 (1996) 81.

[61] R. Siebelink, "The low energy effective action for perturbative heterotic strings on $K_{3} \times T^{2}$ and the $d=4 N=2$ vector-tensor multiplet", hep-th/9709129. 
[62] M. Walton, Phys. Rev. D37 (1987) 377.

[63] S. Hosono, A. Klemm and S. Theisen, Lectures on Mirror Symmetry, from the proceedings of the 3rd Baltic Student Seminar, hep-th/9403096.

[64] P. Candelas, P. Green and T. Hubsch, Phys. Rev. Lett. 62 (1989) 1956.

[65] S. Cecotti, S. Ferrara and L. Girardello, Inter. J. Mod Phys. A, (1989) 2475.

[66] G. Altazabal, A. Font, L. E. Ibanez and F. Quevedo, Nucl. Phys. B461 (1996) 85.

[67] P. S. Aspinwall and M. Gross, Phys. Lett. B382 (1996) 81.

[68] K. Behrndt, G. L Cardoso, B. de Wit, R. Kallosh, D. Lust, T. Mohaupt, Nucl.Phys. B488 (1987) 236.

[69] A. Klemm, W. Lerche and P. Mayr, Phys. Lett. B357 (1995) 313.

[70] S. Hosono, A. Klemm, S. Theisen and S.-T.Yau, Nucl. Phys. B433 (1995) 501.

[71] S. Hosono, A. Klemm, S. Theisen and S.-T.Yau, Com. Math. Phys. 167 (1995) 301.

[72] P. Candelas, X. de la Ossa, A. Font, S. Katz and D.R. Morrison, Nucl. Phys. B416, (1994) 481.

[73] C. Vafa and E. Witten, Nucl. Phys. B (Proc. Suppl.) 46 (1996) 225.

[74] P. Aspinwall and J. Louis, Phys. Lett. B369 (1996) 233; P. Aspinwall, Phys. Lett. B371 (1996) 231, Enhanced gauge symmetries and Calabi-Yau three folds.

[75] D. Morrison and C. Vafa, Nucl.Phys. B473 (1996) 74; Nucl.Phys. B476 (1996) 437.

[76] B. Greene and R. Plesser, Nucl. Phys. B338 (1990) 15.

[77] M. Green, J.H. Schwarz and P.C. West, Nucl. Phys. B254 (1985) 327.

[78] M. Green and J.H. Schwarz, Phys. Lett. 149B (1984) 117.

[79] L. Alvarez-Gaumé and E. Witten, Nucl. Phys. B234 (1984) 269. 
[80] J. Erler, J. Math. Phys. 35 (1994) 1819.

[81] A. Klemm, "On the Geometry behind $N=2$ Supersymmetric Effective actions in four dimensions", hep-th/9705131.

[82] W. Nahm, "On the Seiberg-Witten Approach to electric-magnetic Duality", hep-th/9608121.

[83] I. Antoniadis, E. Gava, K. S. Narain and T. R. Taylor, Nucl. Phys.B455, (1995) 109.

[84] M. Bershadsky, S. Cecotti, H. Ooguri and C. Vafa, Nucl. Phys. B405 (1993) 279 and Comm. Math. Phys. 165 (1994) 311.

[85] E. Witten, Nucl. Phys. B286 (1986) 79.

[86] G. L. Cardoso, G. Curio and D. Lust, Nucl.Phys. B491 (1997) 147.

[87] I. Antoniadis, H. Partouche and T. R. Taylor, "Lectures on Heterotic-Type I duality", hep-th/9706211.

[88] V. Kaplunovsky, Nucl. Phys. B307 (1988) 145, ERRATUM-ibid, B382 (1992) 436.

[89] I. Antoniadis, E. Gava and K. S. Narain, Nucl. Phys. 383 (1992) 109.

[90] V. Kaplunovshy, J. Louis and S. Theisen, Phys. Lett. B357 (1995) 71.

[91] T. Kawai, Phys. Lett. B371 (1996) 191.

[92] T. Kawai, Phys.Lett. B397 (1997) 51.

[93] G. L. Cardoso, G. Curio, D. Lust and T. Mohaupt, Phys.Lett. B382 (1996) 241.

[94] E. Witten, Small instantons in String Theory, Nucl.Phys. B460 (1996) 541.

[95] P. Candelas, G. Horowitz, A. Strominger and E. Witten, Nucl. Phys. B258 (1985) 46.

[96] B. H. Lian and S. T. Yau, Com. Math. Physics. 176 (1996) 163. 
[97] M. Henningson and G. Moore, Nucl.Phys. B482 (1996) 187.

[98] I. Antoniadis and H. Partouche, Nucl.Phys. B460 (1996) 470.

[99] T. Banks and L. Dixon, Nucl. Phys. B307 (1988) 93.

[100] T. Banks and L. Dixon, Nucl. Phys. B307 (1988) 93;

T. Banks, L. Dixon, D. Friedan and E. Martinec, Nucl. Phys. B299 (1988) 613.

[101] A. Giveon, M. Porrati and E. Rabinovici, Phys. Rep. 244 (1994) 77.

[102] S. Cecotti, S. Ferrara and M. Villasante, Int. J. Mod. Phys.A2 (1987) 1839.

[103] T. Kugo and S. Uehara, Nucl. Phys. B222 (1983) 125.

[104] G. L. Cardoso, D. Lust and B. A. Ovrut, Nucl. Phys. B436 (1995) 65.

[105] N. Seiberg, Phys. Lett. B206 (1988)75.

[106] B. de Wit and J. W. van Holten, Nucl. Phys. B155 (1979) 530.

[107] B. de Wit and A. Van Proeyen, Nucl. Phys. B245 (1984) 89;

B.de Wit, P.G. Lawyers, R. Phelippe, Su S.- Q. and A. Van Proeyen, Phys. Lett. 134B (1984) 37.

[108] J. P. Derendinger, S. Ferrara, C. Kounnas and F. Zwigner, Nucl. Phys. B372 (1992) 145.

[109] E. Cremmer, C. Kounnas, A. Van Proyen, J. P. Derendinger, S. Ferrara, B. de Wit and L. Girardello, Nucl. Phys. B250 (1985) 385.

[110] A. Ceresole, R. D’Auria and S. Ferrara, Phys. Lett. B339 (1994) 71; A. Ceresole, R. D’Auria, S. Ferrara and A. Van Proeyen, Nucl. Phys. B444 (1995) 92. 
[111] S. Ferrara and A. Strominger, in Strings '89, eds. R. Arnowitt, R. Bryan, M.J. Duff, D.V. Nanopoulos and C.N. Pope (World Scientific, 1989), p. 245;

A. Strominger, Commun. Math. Phys. 133 (1990) 163;

L.J. Dixon, V.S. Kaplunovsky and J. Louis, Nucl. Phys. B329 (1990) 27;

P. Candelas and X.C. de la Ossa, Nucl. Phys. B355 (1991) 455;

L. Castellani, R. D' Auria and S. Ferrara, Phys. Lett. B241 (1990) 57;

Cl.Q. Grav. 7 (1990) 1767; R. D'Auria, S. Ferrara and P. Fré, Nucl.

Phys. B359 (1991) 705.

[112] S. Ferrara and A. Van Proeyen, Class. Quantum Grav. 6 (1989) L243.

[113] J. Lauer, D. Lüst and S. Theisen, Nucl. Phys. B309 (1988) 771.

[114] N. Seiberg, Nucl. Phys. B303 (1988) 286.

[115] B. Shoeneberg, Elliptic modular functions, Hamburg (1974).

[116] B. de Wit, P. Lauwers and A. Van Proeyen, Nucl. Phys. B255 (1985) 569.

[117] B. de Wit, P.G. Lauwers, R. Philippe, Su,S.-Q.

and A. Van Proeyen, Phys. Lett. 134B (1984) 37.

[118] C. Schoen, Math. Z. 197 (1988) 177.

[119] B. de Wit and A. Van Proeyen, Com. Math. Phys. 149 (1992) 307 Phys. Lett. B293 (1992) 94; B. de Wit, F. Vanderseypen and A. Van Proeyen, Nucl. Phys. B400 (1993) 463.

[120] C. Gomez, R. Hernandez and E. Lopez, Nucl. Phys. B501 (1997) 109.

[121] I. Antoniadis, C. Bachas, C. Fabre, H. Partouche and T. R. Taylor, Nucl. Phys. B498 (1997) 160.

[122] K. Behrndt, G. Lopes Cardoso, B. de Wit, R. Kallosh, D. Lust and T. Mohaupt, Nucl.Phys. B488 (1997) 236.

[123] S. J. Rey, Nucl.Phys. B508 (1997) 569. 